\title{
An efficient approximate moment method for multi-dimensional population balance models - Application to virus replication in multi-cellular systems
}

\author{
Robert Dürr ${ }^{\mathrm{a}, *}$, Thomas Müller ${ }^{\mathrm{a}}$, Stefanie Duvigneau ${ }^{\mathrm{a}}$, Achim Kienle ${ }^{\mathrm{a}, \mathrm{b}}$ \\ ${ }^{a}$ Otto von Guericke University Magdeburg; Universitätsplatz 2, 39106 Magdeburg \\ ${ }^{b}$ Max Planck Institute for Dynamics of Complex Technical Systems; Sandtorstraße 1, 39106 \\ Magdeburg
}

\begin{abstract}
Many particulate processes in process and bioprocess engineering can be described with multi-dimensional population balances. Approximate moment methods are frequently used for their solution. In the present paper a new approach is presented, which is particular efficient when the number of internal coordinates is high. It combines the direct quadrature method of moments with monomial cubatures. With the new method the computational effort increases only polynomially, in the simplest case even only linearly with the number of internal coordinates, compared to an exponential increase for the well known Gausssian cubatures. The technique is evaluated for a five dimensional benchmark problem describing virus replication in continuous cell cultures. Furthermore, the algorithm is applied to analyze influenza virus replication in genetically modified cell lines.

Keywords: multi-dimensional population balance modeling, direct quadrature method of moments, monomial cubature formulas, cell-to-cell variability, influenza virus replication, vaccine production
\end{abstract}

${ }^{*}$ Corresponding author 


\section{Introduction}

Particulate systems are found in a broad field of process engineering applica- 2 tions in which single particles or individuals differ from each other with respect 3 to certain characteristic properties. Examples from chemical processes include agglomeration [1], granulation [2, 3], crystallization [4-6] and coating processes [7]. Further examples for particulate processes are found in bioprocess engineering applications where multi-cellular systems are involved, e.g. cultivation of yeast [8-10], biopolymer production in microorganisms $[11,12]$ and vaccine production processes $[13,14]$. As in the aforementioned examples, nonuniformity of cells with respect to physical properties, like size and shape, but also with respect to intracellular composition is observed. Besides nonuniformity in the process conditions, unsynchronized cell cycles, age distributions [15], stochastic effects on the gene expression level and bistable behaviour on the single cell level [16-18] play a major role in the formation of these variances.

Focus within this contribution is on virus replication in multi-cellular systems which is used for industrial vaccine production processes [13]. The principle process scheme is the following: a cell culture within a bioreactor is inoculated with a low quantity of virus by means of a typical low multiplicity of infection (MOI). The MOI describes the ratio of seed virus to uninfected cells. The seed virions infect the uninfected cells and start to replicate using cellular resources. Synthesized virus is released from the cells to the surrounding medium and can infect still uninfected cells. Thereby, the infection spreads within the cell culture resulting in increasing virus concentration in the medium. The harvested virus is later used for vaccine production. As for other multi-cellular processes cell-to-cell variability has been revealed by flow cytometric analysis [13, 14]. 25 Mathematical models using population balances (see e.g. [19-22]) contribute to 26 a better understanding of the underlying mechanisms and provide a sound basis $\quad 27$ for the design of suitable process intensification and process control schemes. $\quad 28$

In general, two alternative modeling strategies can be followed for the formu- ${ }_{29}$ lation of suitable population balance equations (PBEs). The top down modeling $\quad 30$ 
approach relies on measurements of the cell-to-cell variability by means of a few specific markers which can be measured with sophisticated techniques, e.g. flow 32 cytometry. These are modeled directly and unstructured population balance 33 models are obtained which represent low dimensional integro partial differential 34 equations. Here, global mechanistic kinetics are used to describe the most impor- 35 tant cellular processes. These have to be determined from the experimental data 36 by solving an inverse problem. In our previous work [14, 23] a one-dimensional 37 PBE for influenza A virus replication in MDCK cell cultures was derived and adapted to flow cytometric measurements of intracellular viral nucleoprotein content.

However, interpretation, predictive capacity and accuracy of top down models is limited. Alternatively, a bottom up modeling strategy can be pursued which is based on a detailed description of the single cell kinetics. For influenza vaccine replication such a detailed description features a large number of viral compounds [24]. To account for heterogeneity with respect to the cellular properties, the single particle description is transformed to a structured population balance model. As each particle property translates into an internal coordinate, high dimensional integro partial differential equations are obtained.

Analytic solutions for these PBEs are only found for special cases requiring the application of numerical solution algorithms e.g. discretization based methods like finite volume [25] or finite element methods [26, 27]. Though sophisticated extensions have been developed (see e.g. [28-31]), application is usually limited to low dimensional PBEs with a maximum of three internal coordinates due to increasing computational effort. Alternatively, moment methods can be employed. The basic idea is to track the dynamics of integral quantities of the distribution, so called moments. They are closely related to important properties like mean and variance with respect to the internal coordinates. Usually, those are much easier to interpret than the full number density distributions for most process engineering problems. Furthermore, the number density distribution may be reconstructed from an infinite or even a finite number of its moments [32]. The dynamic moment equations can be derived from the PBE 4 
(see for example [19]) but computation within a closed set of moment equations is only possible for a restricted class of problems.

For most cases, an approximate closure has to be found, e.g. by using the quadrature method of moments (QMOM) where closure is obtained by approximating higher order moments by a weighted sum of abscissas [33]. The QMOM has been applied to a large number of examples from chemical and thermal process engineering (e.g. [31, 34]). Sophisticated extensions for multidimensional PBEs were developed (see e.g. [35-38]). However, due to numerical issues resulting from the underlying solution of generally nonlinear equation systems applications to problems with more than two internal coordinates are rarely found. Alternatively, the direct quadrature method of moments (DQMOM) [39, 40] can be employed. In contrast to QMOM it involves the underlying solution of a system of linear equations.

Besides, computation of the dynamics, the choice of the initial weights and abscissas is of crucial importance. In general, a large number of abscissas is attended by an increased approximation. Yet the numerical effort increases and thus a good balance between the two has to be found. In contrast to onedimensional systems, where Gaussian quadratures offer both, an excellent accuracy and limited computational expenses, the task is much more complicated for multi-dimensional PBEs.

In this contribution we will present an efficient moment approximation technique for multi-dimensional PBEs. The method is based on the DQMOM in combination with monomial cubature rules for the choice of the initial abscissas and weights. These represent a good trade off between numerical effort and approximation accuracy. At first, an analytical solution for the dynamics of weights and abscissas will be derived which is equivalent to our recently presented technique [41]. Application will be demonstrated for virus replication in cell cultures using a generic model formulation. The accuracy of the presented approach is evaluated and compared to other approaches for the choice of initial weights and abscissas. Furthermore, application is shown for the analysis of the impact of host cell heterogeneity on influenza virus replication using genetically modified 
cell lines.

Though focus of this work is on application to bioprocesses, the general algorithm is also suitable for multi-dimensional population balance systems as found e.g. when characterizing the dynamics of shape evolution in crystallization processes $[4,5]$. It will be discussed how the algorithm can be further extended to describe systems in which cell division plays an important role.

\section{Population balance modeling}

Population balance modeling [21] offers a suitable framework to account for the variances in a non-uniform cell ensemble. Neglecting spatial heterogeneity, 101 the dynamics of the corresponding cell number density distribution $n(t, \mathbf{x})$ is 102 characterized by the general population balance equation [21, 42]

$$
\frac{\partial n(t, \mathbf{x})}{\partial t}+\nabla\{\mathbf{G}(\mathbf{t}, \mathbf{x}, \mathbf{c}) n(t, \mathbf{x})\}=-D(t, \mathbf{x}, \mathbf{c}) n(t, \mathbf{x})+P(t, \mathbf{x}, \mathbf{c}) .
$$

Therein, $\mathbf{x}$ represents the vector internal coordinates, e.g. cellular composition, 104 which changes according to the intracellular kinetics $\mathbf{G}$. The right hand side 105 represents sinks and sources resulting from cell death/withdrawal $D$ and other ${ }_{106}$ kinetic processes $P$ like infection in the present paper. Furthermore, the in- 107 tracellular dynamics generally depend on a set of extracellular species c, e.g. ${ }_{108}$ substrates in the medium. Their dynamics are given by

$$
\frac{\mathrm{d} \mathbf{c}}{\mathrm{d} t}=\mathbf{D}_{\mathbf{c}}\left(\mathbf{c}_{i n}-\mathbf{c}\right)+\mathbf{P}_{\mathbf{c}}(\mathbf{c}, \mathbf{F})
$$

where $\mathbf{D}_{\mathbf{c}}$ characterizes the medium exchange and $\mathbf{P}_{\mathbf{c}}$ the integral coupling of ${ }_{110}$ the continuous phase to the cell population

$$
\mathbf{F}=\int_{\mathbf{X}} \mathbf{f}(\mathbf{x}) n(t, \mathbf{x}) \mathrm{d} \mathbf{x} .
$$

\section{Approximate moment dynamics}

In many cases, certain integral quantities of the cell number density distribu- ${ }_{113}$ tion are used to characterize the multi-cellular system. These so called moments $\quad 114$ 
are defined as

$$
m_{l_{1}, \ldots, l_{N_{d}}}=\int_{\mathbf{X}} x_{1}^{l_{1}} \ldots x_{N_{d}}^{l_{N_{d}}} n(t, \mathbf{x}) \mathrm{d} \mathbf{x}
$$

and are directly related to important properties of the overall cell number density $\quad 116$ distribution, like the overall number of particles and mean with respect to an 117 arbitrary particle property $x_{k}$. The corresponding dynamic moment equations $\quad 118$ are derived from combination of (1) and (4)

$$
\begin{aligned}
\frac{\mathrm{d}}{\mathrm{d} t} m_{l_{1}, \ldots, l_{d}} & =-\sum_{k=1}^{N_{d}} \int_{\mathbf{X}} x_{1}^{l_{1}} \ldots x_{N_{d}}^{l_{N_{d}}} \frac{\partial}{\partial x_{k}}\left\{g_{k} n\right\} \mathrm{d} \mathbf{x} \\
& +\int_{\mathbf{X}} x_{1}^{l_{1}} \ldots x_{N_{d}}^{l_{N_{d}}}(P-D n) \mathrm{d} \mathbf{x} .
\end{aligned}
$$

However, a closed set of equations for the moment dynamics can only be found $\quad 120$ for special classes of $\mathbf{G}, D$ and $P$. Applying the DQMOM [39], it is assumed ${ }_{121}$ that $n(t, x)$ can be represented by a weighted sum of $N_{\alpha}$ delta functions. Hence, ${ }_{122}$ integral quantities can be approximated by

$$
\int_{\mathbf{X}} f(\mathbf{x}) n(t, \mathbf{x}) \mathrm{d} \mathbf{x} \approx \sum_{\alpha=1}^{N_{\alpha}} f\left(\mathbf{x}_{\alpha}(t)\right) w_{\alpha}(t)=\sum_{\alpha=1}^{N_{\alpha}} f_{\alpha} w_{\alpha}
$$

with $w_{\alpha}$ being the weights and $\mathbf{x}_{\alpha}$ the abscissas. Instead of tracking the mo- ${ }_{124}$ ment dynamics focus is on the temporal evolution of the weights and weighted 125 abscissas

$$
\frac{\mathrm{d} w_{\alpha}}{\mathrm{d} t}=a_{\alpha}, \quad \frac{\mathrm{d} w_{\alpha} \mathbf{x}_{\alpha}}{\mathrm{d} t}=\mathbf{b}_{\alpha} .
$$

These are derived as follows. At first, the approximation (6) is combined with ${ }_{127}$ (5) and (7), which yields

$$
\begin{aligned}
& \sum_{\alpha=1}^{N_{\alpha}}\left\{\left(1-\sum_{k=1}^{N_{d}} l_{k}\right) x_{1, \alpha}^{l_{1}} \ldots x_{N_{d}, \alpha}^{l_{N_{d}}} a_{\alpha}+\right.
\end{aligned}
$$

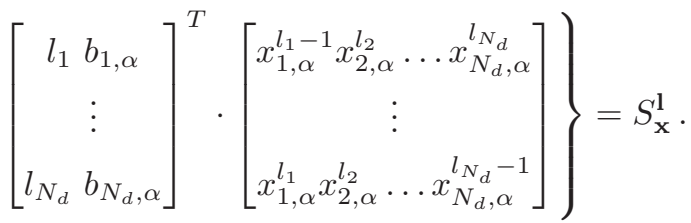


Here, $S_{\mathbf{x}}^{\mathbf{l}}$ contains the right hand side of (5) and can also be approximated ${ }^{129}$ using (6). The previous equation is evaluated for $\left(N_{d}+1\right) N_{\alpha}$ distinct moments $\quad{ }_{130}$ resulting in a linear system

$$
A \cdot\left(\begin{array}{l}
\mathbf{a} \\
\mathbf{b}
\end{array}\right)=\mathbf{S}_{\mathbf{x}}^{\mathbf{l}}
$$

which is then solved for the unknown right hand side expressions of (7).

Note, that the choice of the moments for the construction of the linear system $\quad{ }_{133}$ is of crucial importance for the overall performance of the moment approxima- ${ }_{134}$ tion. Moments related to important quantities such as the zeroth order moment $\quad{ }_{135}$ (overall number of particles) and the first order moments (related to mean val- ${ }^{136}$ ues) should always be included. The remaining moments have to be chosen with $\quad{ }_{137}$ care as some choices may lead to a poor approximation performance or even to $\quad 138$ singular matrices [40]. Furthermore, $A$ resembles a van der Monde matrix and ${ }_{139}$ can become ill conditioned easily, in particular for a large number of abscissas $\quad{ }_{140}$ and if two or more abscissas are close to each other in the state space, respec- ${ }_{141}$ tively. To overcome these problems different methods have been suggested, like $\quad 142$ the application of fractal moments [43]. Alternatively, numerical problems can ${ }_{143}$ be circumvented employing an analytical solution of (9). 144

In case of $P=0$ such an analytical solution can be derived without even ${ }_{145}$ using the matrix formulation (9) [44]. For zeroth order moment (8) is simplified $\quad 146$ to

$$
\sum_{\alpha=1}^{N_{\alpha}} a_{\alpha}=-\sum_{\alpha=1}^{N_{\alpha}} w_{\alpha} D_{\alpha}
$$

and by comparing the coefficients the weight dynamics are given as

$$
\frac{\mathrm{d} w_{\alpha}}{\mathrm{d} t}=-w_{\alpha} D_{\alpha}
$$

In a similar procedure, (8) can be used for arbitrary first order moments

$$
\sum_{\alpha=1}^{N_{\alpha}} b_{k, \alpha}=\sum_{\alpha=1}^{N_{\alpha}} w_{\alpha}\left(g_{k, \alpha}-x_{k, \alpha} D_{\alpha}\right), \quad k=1, \ldots, N_{d}
$$


to derive the dynamics of the weighted abscissas

$$
\frac{\mathrm{d} w_{\alpha} x_{k, \alpha}}{\mathrm{d} t}=b_{k, \alpha}=w_{\alpha}\left(g_{k, \alpha}-x_{k, \alpha} D_{\alpha}\right), \quad k=1, \ldots, N_{d} .
$$

Combination of the last two relations yields

$$
\frac{\mathrm{d} \mathbf{x}_{\alpha}}{\mathrm{d} t}=\mathbf{G}_{\alpha}
$$

which also means that the abscissas move along the characteristic curves of 152 (1) and the DQMOM reduces to a method of characteristics for $P=0$. For ${ }^{153}$ introduction to characteristics we refer to [45]. It has to be mentioned, that even $\quad 154$ if these relations are derived from zeroth and first order moments, conservation $\quad 155$ of higher (and fractal) order moments is also guaranteed by means of fulfilling 156 (8) for arbitrary l. For the two dimensional case this shown in the Appendix. In ${ }_{157}$ [41] the same results for the dynamics of abscissas and weights (denoted scaling $\quad 158$ factors) have been obtained based on a scaled PBE.

\section{Efficient choice of abscissas and weights}

In the previous section the dynamics of abscissas and weights were derived. ${ }^{161}$ However, so far no word was lost about the initial values for the corresponding $\quad 162$ ODEs (11) and (14). In the DQMOM context initial abscissas and weights are 163 chosen based on moments (or more general integral quantities) of the initial 164 number density distribution.

$$
\begin{aligned}
m_{l_{1}, \ldots, l_{N_{d}}}(t=0) & =\int_{\mathbf{X}} x_{1}^{l_{1}} \cdots x_{N_{d}}^{l_{N_{d}}} n(t=0, \mathbf{x}) \mathrm{d} \mathbf{x} \\
& \approx \sum_{\alpha=1}^{N_{\alpha}} w_{\alpha}(t=0) x_{1, \alpha}^{l_{1}}(t=0) \cdots x_{\alpha, N_{d}}^{l_{N_{d}}}(t=0) .
\end{aligned}
$$

The choice of initial weights and abscissas represents an important factor in the $\quad{ }_{166}$ performance of the overall moment approximation algorithm. For the following $\quad 167$ explanations it is assumed that the initial distribution is normalized

$$
\int_{\mathbf{X}} n(t=0, \mathbf{x}) \mathrm{d} \mathbf{x}=\int_{\mathbf{X}} n_{0}(\mathbf{x}) \mathrm{d} \mathbf{x}=1 .
$$


In general, a larger number of abcissas $N_{\alpha}$ comes along with an increased ap- $\quad 169$ proximation accuracy but also with an increased computational effort as the 170 number of ODEs for a DQMOM approximation of the moments increases. In 171 standard literature on numerical integration (see e.g. [46, 47]) a large number of $\quad 172$ cubature formulas is found which can be roughly classified into random based 173 and deterministic rules, where the latter contain product rules and non product $\quad 174$ rules. The abscissas and weights are only computed once at the beginning of the $\quad 175$ overall algorithm. Thus, computational effort of this step is negligible compared 176 to the numerical effort for the solution of the weight and abscissa dynamics. $\quad 177$

When applying random based rules, abscissas are determined by random 178 sampling of the integration region. The corresponding numerical integration 179 method is also known as Monte Carlo integration [46]. Its popularity is based on 180 the straightforward generation of abscissas and it is commonly used to generate 181 reference solutions. However, in general a large number of samples is necessary $\quad 182$ and thus overall computational effort is unreasonable large. 183

Product rules are multi-dimensional extensions of one-dimensional quadra- ${ }_{184}$ ture rules. In the one-dimensional case, Gaussian quadrature rules which are 185 based on orthogonal polynomials can be applied to come up with appropriate $\quad 186$ sets of abscissas and weights, e.g. if the initial distribution corresponds to a 187 Gaussian distribution, the Gauss Hermite rule may be applied [46]. These for- ${ }^{188}$ mulas can be extended to multi-dimensional problems by using tensor products 189 of one-dimensional weight and abscissa sets. In consequence, those rules suffer 190 badly from the curse of dimensionality, as the sizes of the weight and abscissa 191 sets generally increase exponentially with the number of dimensions. This is a 192 major disadvantage for high dimensional applications. 193

Alternatively, the set of abscissas is generated directly in the full property ${ }_{194}$ state space instead of tensoring using non product formulas. Their basic idea 195 is to exploit special properties of $n_{0}(t, \mathbf{x})$ to come up with abscissa and weight $\quad 196$ sets that scale polynomially, in the best case even linearly with $N_{d}$. A derivation 197 based on generator functions can be found in [48]. Those rules are also denoted 198 as monomial cubature rules. 
One of the most renowned formulas are sigma point formulas as introduced 200 by Julier and Uhlmann (see e.g. $[49,50]$ ). They are commonly applied for the un- 201 scented Kalman filter for state and parameter estimation. For a $N_{d}$ dimensional 202 Gaussian distribution $\mathcal{N}(\mu, \boldsymbol{\Sigma})$ the abscissas and weights can be determined by ${ }_{203}$

$$
\begin{array}{cccc}
x_{0}= & \mu & w_{0}= & \frac{\lambda}{\lambda+N_{d}} \\
x_{i}= & \mu+\sqrt{\lambda+N_{d}} \sqrt{\Sigma_{i}} & w_{i}= & \frac{1}{2\left(\lambda+N_{d}\right)} \\
x_{N_{d}+i}= & \mu-\sqrt{\lambda+N_{d}} \sqrt{\Sigma_{i}} & w_{N_{d}+i}= & \frac{1}{2\left(\lambda+N_{d}\right)}
\end{array}
$$

where the tuning parameter $\lambda$ is given by

$$
\lambda=\widetilde{\alpha}^{2}\left(\kappa-N_{d}\right)+N_{d}
$$

and controls the spread of the abscissas in the state space and $\sqrt{\boldsymbol{\Sigma}}_{i}$ is the i-th 205 column of the covariance matrix square root. Here, $\kappa$ and $\widetilde{\alpha}$ are additional tuning $\quad 206$ parameters [50]. The size of the abscissa set scales linearly with dimension, which 207 is a crucial advantage in particular for high dimensional applications. To improve 208 the accuracy higher order non product rules have been suggested, e.g. in [51], 209 which do scale polynomially with dimension. 210

If the assumption of an Gaussian initial distribution is not justified, the sigma ${ }^{211}$ point approach has to be modified. For some types of the initial distribution 212 special transformation formulas exist. For example if $n_{0}$ corresponds to a one- ${ }^{213}$ dimensional logarithmic normal distribution $\mathcal{L}\left(\mu_{\mathcal{L}}, \sigma_{\mathcal{L}}\right)$ the transformed set can ${ }^{214}$ be computed as follows [52] 215

1. sigma points $\mathbf{x}_{i}$ are computed for $\mathcal{N}(0,1)$ according to $(17) \quad 216$

2. the transformed sigma point set is then given by 217

$$
x_{i, \mathcal{L}}=\exp \left(\mu_{\mathcal{L}}+\sigma_{\mathcal{L}_{k}} x_{i}\right), \quad i=1, \ldots, N_{\alpha}
$$

3. the set of weights is not changed within the transformation procedure and ${ }_{218}$ is given by (17).

Further examples for those transformation formulas include $\gamma$ - and $t$-Student 220 distributions [52]. 
Yet, no general transformations exists if $n_{0}$ is multi modal. To improve this 222 situation a two step procedure is advantageous, which was applied in [53] to 223 approximate uncertainties of biological models. At first, $n_{0}$ is approximated by 224 a weighted sum of Gaussian distributions

$$
n_{0}(\mathbf{x}) \approx \sum_{k=1}^{N_{\mathrm{GMD}}} w_{k}^{\mathrm{GMD}} \mathcal{N}\left(\mu_{k}, \boldsymbol{\Sigma}_{k}\right)
$$

This approximation is also termed Gaussian mixed density (GMD) in some 226 references. Afterwards, abscissas and weights are determined for each Gaussian 227 applying the standard sigma point formula. For the overall approximation of 228 the integrals the general formula (6) has to be adapted

$$
\int_{\mathbf{X}} f(\mathbf{x}) n_{0}(\mathbf{x}) \mathrm{d} \mathbf{x} \approx \sum_{k=1}^{N_{\mathrm{GMD}}} w_{k}^{\mathrm{GMD}} \sum_{\alpha=1}^{N_{\alpha}} w_{k, \alpha} f\left(\mathbf{x}_{k, \alpha}\right) .
$$

The overall number of weights and abscissas is now given by $N_{\mathrm{GMD}} N_{\alpha}$. For ${ }_{230}$ more complex distributions (e.g. multi modal on a logarithmic scale) the previ- ${ }^{231}$ ously mentioned approaches can be combined to come up with more accurate 232 approximations. 233

In the following, the different approaches will be evaluated for a generic ${ }_{234}$ benchmark problem describing virus replication in a multi-cellular system. Fur- 235 thermore, the technique will be applied to a high dimensional model which ${ }_{236}$ characterizes the spread of influenza A virus in a cell culture.

\section{Application to a simple model of virus replication in cell cultures 238}

\subsection{Model formulation 239}

The presented model is adapted from [54] and comprises the key elements of $\quad 240$ a viral replication process within a host cell. The general replication mechanism 241 is depicted in Fig. 1. Virus particles bind to the surface of uninfected cells. After 242 a virus particle has passed the cell membrane, the virus genome is uncoated and 243 thus a certain amount of viral genetic information $[\mathrm{gen}]$ is injected to the cell. 244 From this, a viral genomic template $[t e m]$ is produced. It delivers the blueprints 245 


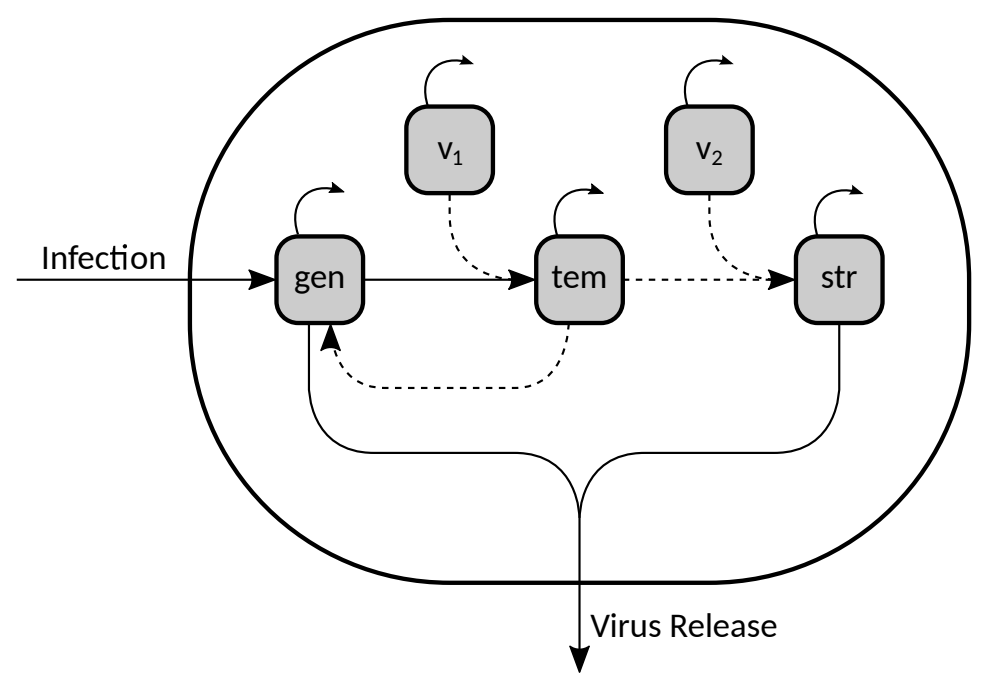

Figure 1: Scheme of the assumed intracellular mechanisms for viral replication (gen: viral genome, tem: viral genome template, str: viral structural protein, $v_{1} \& v_{2}$ : enzymes)

for the replication of further viral genomic template and viral structural protein $\quad 246$ $[s t r]$. It is assumed, that the corresponding production rates are catalyzed by 247 intracellular enzymes $\left[v_{1}\right]$ and $\left[v_{2}\right]$. It has to be mentioned that in addition to the 248 original model proposed by Haseltine and coworkers [54, 55] a degradation of the 249 virus genome template and the structural protein is modeled. Virus progeny is 250 formed by binding of structural protein and viral genome. In a final step mature 251 virus is released from the cell. Balancing of the species yields the following 252 dynamics on the single cell level described by the following coupled system of 253 ODEs

$$
\dot{\mathbf{x}}=\frac{\mathrm{d}}{\mathrm{d} t}\left(\begin{array}{c}
{[\text { tem }]} \\
{[\mathrm{gen}]} \\
{[s t r]} \\
{\left[v_{1}\right]} \\
{\left[v_{2}\right]}
\end{array}\right)=\mathbf{h}=\left(\begin{array}{c}
k_{1}\left[v_{1}\right][\mathrm{gen}]-k_{6}[\mathrm{tem}] \\
k_{3}[\mathrm{tem}]-k_{1}\left[v_{1}\right][\mathrm{gen}]-k_{5}[\mathrm{gen}][\mathrm{str}]-k_{7}[\mathrm{gen}] \\
k_{2}[\mathrm{tem}]\left[v_{2}\right]-k_{4}[\mathrm{str}]-k_{5}[\mathrm{gen}][\mathrm{str}] \\
f_{v_{1}} \\
f_{v_{2}}
\end{array}\right)
$$


where $f_{v_{1}}$ and $f_{v_{2}}$ are the enzyme degradation and production rates. By this 255 means the analysis of host cell resource limitations on the process can be facil- 256 itated, see [56]. To transform the description to the macroscopic scale within 257 the framework of population balance modeling, the single cell states directly 258 translate into internal coordinates of a corresponding PBE. The dynamics of 259 the number density distribution of infected cells is thus given by

$$
\frac{\partial i_{c}(t, \mathbf{x})}{\partial t}=\underbrace{-\nabla_{\mathbf{x}}\left\{\mathbf{h}(\mathbf{x}) i_{c}(t, \mathbf{x})\right\}}_{\text {intracellular reactions }}+\underbrace{k_{\mathrm{inf}} U_{c}(t) V(t) \mathcal{I}(\mathbf{x})}_{\text {infection }}-\underbrace{k_{\mathrm{cd}, i_{c}}(\mathbf{x}) i_{c}(t, \mathbf{x})}_{\text {cell death }} .
$$

Here, it is assumed, that infected cells are "produced" by binding of free virus 261 particles $V$ to uninfected cells $U_{c}$. At this point cell-to-cell variability for newly 262 infected cells is taken into account by distributing infected cells into the space ${ }_{263}$ of internal properties according to a normal distribution

$$
\mathcal{I}(\mathbf{x})=\mathcal{N}(\mu, \mathbf{\Sigma})
$$

with mean $\mu$ and covariance $\Sigma$. At this point, it has to be mentioned that the ${ }^{265}$ special case when the population becomes stochastic due to a low number of 266 virus particles or uninfected cells as described in [57] is not considered in the 267 present publication. It is thereby assumed, that stochastic fluctuations of the 268 expected number of newly infected cells $k_{\text {inf }} U_{c}(t) V(t)$ are neglected. 269

In result of the infection, cells undergo apoptosis and lysis. These cellular 270 processes are summarized as "cell death" and are represented by a cell death 271 coefficient $k_{\mathrm{cd}, i_{c}}(\mathbf{x})$. Its functional dependency on the vector of internal coor- 272 dinates is motivated by the common assumption, that apoptosis and lysis are 273 commonly induced by specific viral proteins.

In contrast to infected cells, uninfected cells $U_{c}$ are not differentiated with re- $\quad 275$ spect to their intracellular composition. In result, their dynamics is represented 276 by the following ODE

$$
\frac{\mathrm{d} U_{c}(t)}{\mathrm{d} t}=-k_{\mathrm{inf}} U_{c}(t) V(t)+k_{\mathrm{gro}, U_{c}} U_{c}(t)-k_{\mathrm{cd}, U_{c}} U_{c}(t) .
$$


Table 1: Parameter values for adapted Haseltine model (22) - (27)

\begin{tabular}{cccccc}
\hline Parameter & Value & \multicolumn{5}{c}{ Parameter } & Value \\
\hline$k_{1}$ & $3.1310^{-4}$ & $\#^{-1} t u^{-1}$ & $k_{\text {inf }}$ & $110^{-8}$ & $\frac{\text { [Vol] }^{2}}{\text { cells virions } t u}$ \\
$k_{2}$ & $25.0010^{-0}$ & $\#^{-1} t u^{-1}$ & $k_{\mathrm{cd}, i_{c}}$ & $510^{-3}$ & $t u^{-1}$ \\
$k_{3}$ & $7.0010^{-1}$ & $t u^{-1}$ & $k_{\mathrm{cd}, U_{c}}$ & $1.510^{-3}$ & $t u^{-1}$ \\
$k_{4}$ & $2.0010^{-0}$ & $t u^{-1}$ & $k_{\mathrm{gro}, U_{c}}$ & $110^{-3}$ & $t u^{-1}$ \\
$k_{5}$ & $7.5010^{-6}$ & $\#^{-1} t u^{-1}$ & $k_{\mathrm{deg}, V}$ & $910^{-1}$ & $t u^{-1}$ \\
$k_{6}$ & $1.0010^{-1}$ & $t u^{-1}$ & & & \\
$k_{7}$ & $1.4010^{-1}$ & $t u^{-1}$ & & & \\
\hline
\end{tabular}

Therein, $k_{\text {gro }} U_{c}(t)$ and $k_{\mathrm{cd}, U_{c}} U_{c}(t)$ characterize the growth and cell death rates $\quad 278$ of the uninfected cells, respectively.

At the end of a successful replication, virus particles are released from the in- $\quad 280$ fected cells to the medium with individual rates. These depend on the particular ${ }_{281}$ intracellular state of each cell and is given by

$$
r_{\text {rel }}(\mathbf{x})=k_{5}[\text { gen }][s t r] .
$$

according to the single cell dynamics.

In addition to the dynamics of the cell species, the dynamics of the virus particles in the medium has to be taken into account. For this ideal mixing is assumed. The overall dynamics of active virus particles are thus determined by the following ordinary differential equation

$$
\frac{\mathrm{d} V(t)}{\mathrm{d} t}=\int_{\mathbf{X}} r_{\mathrm{rel}}(\mathbf{x}) i_{c}(t, \mathbf{x}) \mathrm{d} \mathbf{x}-k_{\mathrm{inf}} U_{c}(t) V(t)-k_{\mathrm{deg}, V} V(t)
$$

where the rate coefficient $k_{\mathrm{deg}}$ characterizes the degradation and inactivation of $\quad 288$ free virus particles. The integral term on the right hand side represents the virus 289 release of all individual cells. The simulation results shown in the following are 290 based on the set of parameter values given in Table 1. Therein, $t u$ represents 291 time units. 


\subsection{Implementation details}

All cubatures are directly derived from the initial distribution of the infected 294 cells $\mathcal{N}(\mu, \boldsymbol{\Sigma})$ to obtain the initial abscissas and weights. For the subsequent 295 benchmark, five different cubatures have been implemented as representatives 296 of the above mentioned cubature groups: mean approximation ( $\mathrm{ME}, N_{\alpha, \mathrm{ME}}={ }^{297}$ 1 ), sigma point formula ( $\left.\mathrm{SP}, N_{\alpha, \mathrm{SP}}=11\right)$, higher order non product formula ${ }_{298}$ $\left(\mathrm{HONP}, N_{\alpha, \mathrm{HONP}}=51\right)$, Gaussian mixed density approach $\left(\mathrm{GMD}, N_{\alpha, \mathrm{GMD}}={ }^{299}\right.$ 110) and Gaussian product formula $\left(\mathrm{GA}, N_{\alpha, \mathrm{GA}}=243\right)$ which are all evaluated 300 against an Monte Carlo integration $\left(\mathrm{MC}, N_{\alpha, \mathrm{MC}}=10^{4}\right)$. A detailed description ${ }_{301}$ of rules and comments on the numerical effort in terms of size of the overall 302 ODE system which has to be solved is given in the supplemetary information. $\quad 303$

\subsection{Single infection cycle}

At first, a single infection cycle scenario is considered. Here, it is assumed 305 that all cells are infected initially and no free virus is present in the medium at 306 the beginning of the process. Thus, the initial conditions are given by

$$
\begin{aligned}
& V(t=0)=0 \frac{\text { virions }}{[\mathrm{Vol}]}, \quad U_{c}(t=0)=0 \frac{\text { cells }}{[\mathrm{Vol}]}, \\
& I_{c}(t=0)=I_{c, 0} \frac{\text { cells }}{[\mathrm{Vol}]} .
\end{aligned}
$$

Thereby, any observed cellular heterogeneity would be an effect of initial cell-to- 308 cell variability while heterogeneity resulting from the delayed infection process 309 is negligible. For this reason, a similar experimental setup is often used to de- 310 termine single cell parameters.

Vice versa, this setup is also very useful to analyze the performance of the 312 presented moment approximation algorithm. As the infection process is negligi- ${ }^{313}$ ble, the dynamics of the corresponding infected cell number density distribution $\quad 314$ is now given by

$$
\frac{\partial i_{c}(t, \mathbf{x})}{\partial t}=-\nabla_{\mathbf{x}}\left\{\mathbf{h}(\mathbf{x}) i_{c}(t, \mathbf{x})\right\}-k_{\mathrm{cd}, i_{c}}(\mathbf{x}) i_{c}(t, \mathbf{x})
$$

with initial condition following from the above representations

$$
i_{c}(t, \mathbf{x})=\mathcal{I}(\mathbf{x})=I_{c, 0} \mathcal{N}(\mu, \boldsymbol{\Sigma}),
$$


where

$$
\mu=[1,10,1,80,40]^{\mathrm{T}}, \quad \boldsymbol{\Sigma}=0.05 \operatorname{diag}\left(\mu^{2}\right)
$$

Furthermore, the dynamics of the free virions in the medium is defined by (27). 318

For the given setup, the initial number of infected cells is normalized to $I_{c, 0}={ }_{319}$ 1. Thus, in the following, all values can be viewed as normalized quantities. Now, arbitrary integral quantities of the infected cell number density with respect to 321 the internal coordinates can be approximated by (6)

$$
\int_{\mathbf{X}} f(\mathbf{x}) i_{c}(t, \mathbf{x}) \mathrm{d} \mathbf{x} \approx \sum_{\alpha=1}^{N_{\alpha}} w_{\alpha}(t) f\left(\mathbf{x}_{\alpha}(t)\right) .
$$

In accordance to the derivation presented previously, the dynamics of the weights 323 and abscissas are given by

$$
\dot{\mathbf{x}}_{\alpha}(t)=\frac{\mathrm{d}}{\mathrm{d} t}\left(\begin{array}{c}
{[\mathrm{tem}]_{\alpha}} \\
{[\mathrm{gen}]_{\alpha}} \\
{[s t r]_{\alpha}} \\
{\left[v_{1}\right]_{\alpha}} \\
{\left[v_{2}\right]_{\alpha}}
\end{array}\right)=\mathbf{h}\left(\mathbf{x}_{\alpha}\right)
$$

and

$$
\dot{w}_{\alpha}(t)=k_{\mathrm{cd}, i_{c}}\left(\mathbf{x}_{\alpha}\right) w_{\alpha}(t) .
$$

The technique presented in the previous section can be applied directly to ${ }_{326}$ approximate integral quantities from the number density distribution includ- 327 ing virus release rate (26) and moments with respect to the intracellular states. 328 Within this setup the performance of different cubature formulas will be ana- ${ }_{329}$ lyzed. A random based cubature rule with $N_{\mathrm{MC}}=10^{4}$ is used as a reference ${ }_{330}$ solution. As the abscissas move along the characteristic curves of the PBE, this 331 corresponds to a Monte-Carlo evaluation of the full solution achieved with the ${ }_{332}$ method of characteristics (see also [41, 58]). 333

In a first scenario, it is assumed, that the cell death does not depend on the in- $\quad{ }_{334}$ tracellular composition. An example for this setup can be found when analyzing 335 


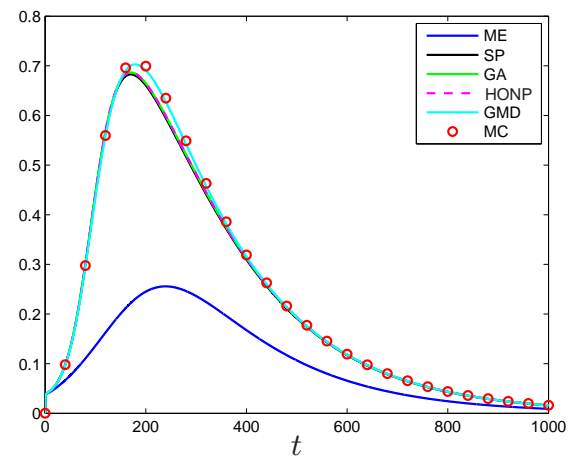

(a) Virus release rate

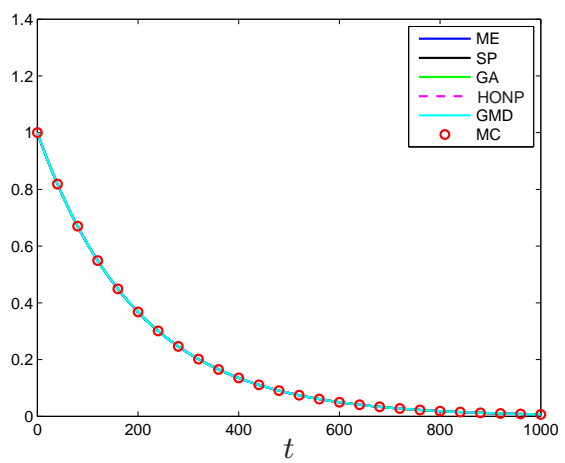

(b) Infected cells

Figure 2: Comparison of the virus release rate integral and the overall number of infected cells for different cubatures

the effects of physical or chemical stimuli on the cell, like medium temperature, 336 radiation or $\mathrm{pH}-$ level which effect all cells in the same manner. Furthermore, the ${ }^{337}$ enzyme levels remain constant in course of the infection process. In consequence, $3 з 8$ the corresponding rate coefficients are given by

$$
k_{\mathrm{cd}, i_{c}}(\mathbf{x})=k_{\mathrm{cd}, i_{c}}, \quad f_{v_{1}}=f_{v_{2}}=0 .
$$

In Fig. 2(a) the temporal change of the virus release rate is depicted for all 340 previously introduced abscissa formulas. It can be seen that most formulas reach $\quad 341$ a good approximation accuracy with the GMD approach slightly outperforming 342 the other approaches. In contrast, an approximation which uses only one abscissa $\quad 343$ located at the mean of the initial distribution exhibits an significant error. In 344 Fig. 2(b) the approximations of the zeroth order moment of $i_{c}(t, \mathbf{x})$ (i.e. the ${ }_{345}$ overall number of infected cells)

$$
I_{c}(t)=m_{\mathbf{0}}(t)=\int_{\mathbf{X}} i_{c}(t, \mathbf{x}) \mathrm{d} \mathbf{x}
$$

is depicted. It can be seen, that all cubature formulas yield an similar accuracy. 347 In contrast, different approximation accuracies are observed for the approxima- 348 

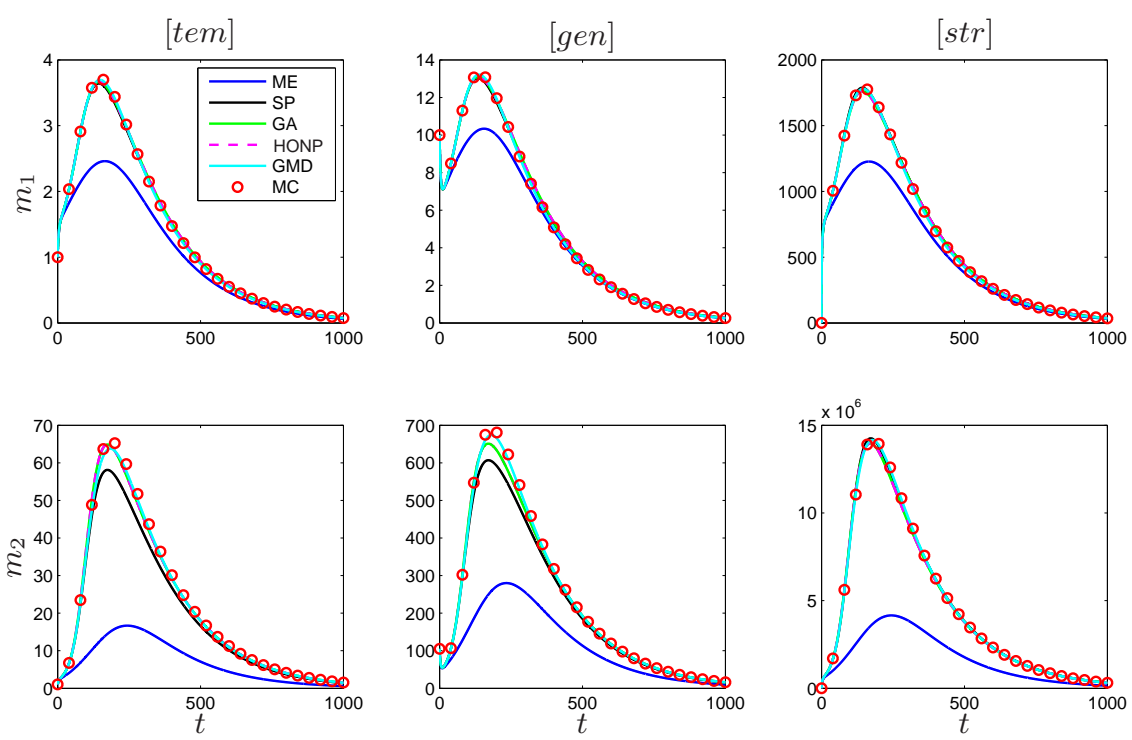

Figure 3: First and second order pure moments of the distribution for different cubatures

tion of the first and second order pure moments

$$
\begin{aligned}
& m_{1, x_{k}}(t)=\int_{\mathbf{X}} x_{k} i_{c}(t, \mathbf{x}) \mathrm{d} \mathbf{x} \\
& m_{2, x_{k}}(t)=\int_{\mathbf{X}} x_{k}^{2} i_{c}(t, \mathbf{x}) \mathrm{d} \mathbf{x}
\end{aligned}
$$

which can be seen in Fig. 3. Similar to the approximation of the overall virus 350 release integral, good accuracy is obtained for all approaches except the ME for- 351 mula (highlighted with a blue curve) for the approximation of the first moments. 352 However, the GMD approach (cyan) outperforms the other formulas with a max- 353 imum relative error around 1\%. The maximum errors of the HONP, SP and GA 354 approaches are of similar values. In contrast, the SP approach exhibits larger 355 errors than the other approaches (apart from the ME approach). In particular, 356 this can be seen for the second order moment with respect to $[\mathrm{tem}]$ where the 357 maximum relative error is around 10\%. Again, the GMD approach outperforms 358 the other ones.

In the second scenario, it is assumed that each cell is characterized by an 360 


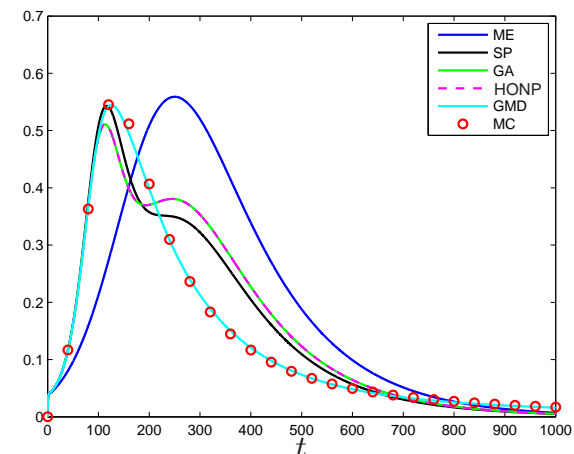

(a) Virus release rate integral

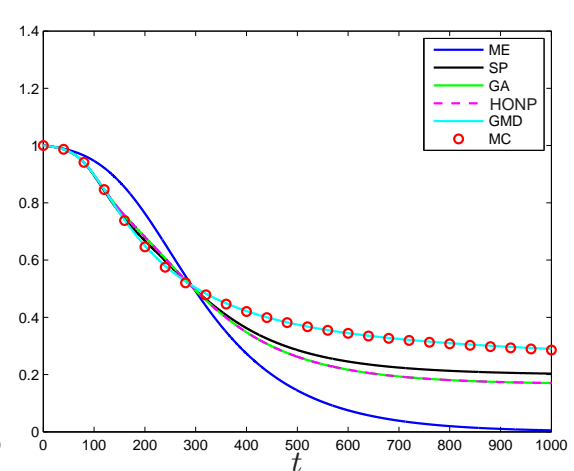

(b) Infected cells

Figure 4: Overall virus release rate integral and overall number of infected cells for different cubatures

individual cell death rate depending on the intracellular composition

$$
k_{\mathrm{cd}, i_{c}}(\mathbf{x})=k_{\mathrm{cd}, i_{c}} r_{\mathrm{rel}}(\mathbf{x}), \quad f_{v_{1}}=f_{v_{2}}=0 .
$$

This means, that cells which are characterized by an increased intracellular 362 amount of viral compounds $[$ gen $]$ and $[s t r]$ have a lower survival probability 363 than cells that are characterized by a low amount of these. Examples for such 364 behavior are found for example for apoptosis induction [18, 59]. Apoptosis is 365 one form of programmed cell death and is for example induced as a reaction to 366 environmental stress or the viral infection.

Approximations of the integral virus release for the different approaches 368 can be seen in Fig. 4(a). It can be observed, that neither the standard sigma 369 point approach, nor the higher order non product cubature approach and the 370 Gaussian approximation are able to approximate the overall virus release rate 371 with a reasonable accuracy. In contrast, the GMD approach shows good results. 372 The corresponding relative error stays within narrow bounds. The same is also 373 observed from Fig. 4(b) which shows the overall number of infected cells. Apart 374 from the approximation using the mean of the distribution as a sole abscissa, 375 all other approaches show at least a sufficient performance up to $300 \mathrm{tu}$. Again, 376 the GMD approach outperforms the other approaches and stays within very 377 narrow error bounds. In Fig. 5 approximations of the first and second order 378 

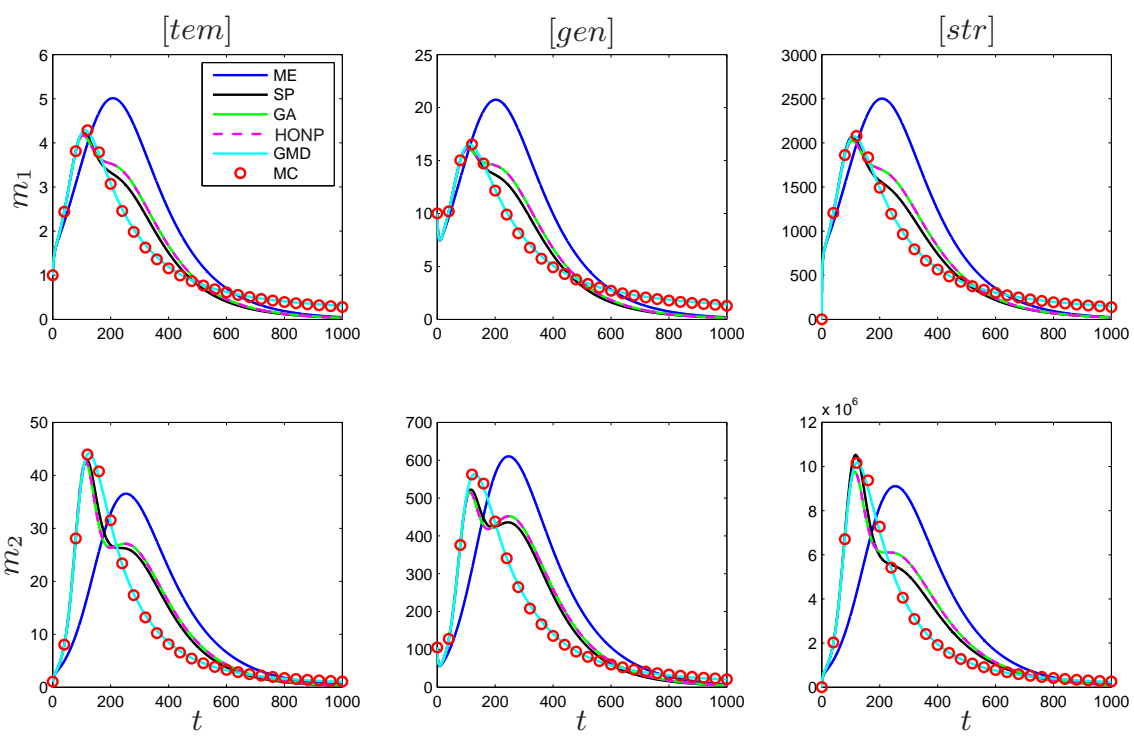

Figure 5: First and second order pure moments of the distribution for different cubatures

moments are depicted. As for the approximations of the overall cell number and 379 the overall virus release, all approaches other than the GMD approach show 380 significant approximation errors.

\subsection{Multiple infection cycles}

382

In the previous scenario, it was assumed, that all cells are infected initially. 383 However, in real vaccine production processes, the initial MOI is generally low: 384 A low number of virus particles is used to inoculate the reactor to obtain a 385 large harvest of replicated virus particles. In the following, the accuracy of the 386 approximation approaches presented in the previous section will be shown for 387 this multiple infection cycles scenario.

In contrast to the previous scenario, now initially only virions and uninfected $\quad 389$ cells are present and thus the initial conditions are given by

$$
U_{c}(t=0)=10^{8} \frac{\text { cells }}{[\mathrm{Vol}]}, \quad V(t=0)=10^{3} \frac{\text { virions }}{[\mathrm{Vol}]}, \quad I_{c}(t=0, \mathbf{x})=0 \frac{\text { cells }}{[\mathrm{Vol}]} .
$$

In contrast to the single infection cycle setup, the full PBE (23) has to be taken 391 into account to characterize the infected cell dynamics. In the DQMOM context, 392 
this would require the numerical solution of (9) which may result in numerical 393 difficulties. As an alternative, the overall problem is reformulated as a series of 394 initial value problems

$$
\frac{\partial i_{c, k}(t, \mathbf{x})}{\partial t}=-\nabla_{\mathbf{x}}\left\{\mathbf{h}(\mathbf{x}) i_{c, k}(t, \mathbf{x})\right\}-k_{\mathrm{cd}, i_{c}}(\mathbf{x}) i_{c, k}(t, \mathbf{x})
$$

with initial conditions given by

$$
i_{c, k}\left(t=t_{k}, \mathbf{x}\right)=\underbrace{k_{\text {inf }} U_{c}\left(t_{k-1}\right) V\left(t_{k-1}\right)\left(t_{k}-t_{k-1}\right)}_{\text {Inf }\left(t_{k}\right)} \mathcal{I}(\mathbf{x}) .
$$

Thereby, each of the initial value problems describes the dynamics of cells 397 $i_{c, k}(t, \mathbf{x})$ which have been infected in the interval $\left[t_{k-1}, t_{k}\right)$. Comparing the 398 obtained series reformulation to the single cycle infection formulation (29), it 399 can be seen that the reformulated multiple infection cycles correspond to finite $\quad 400$ number of single infection cycle scenarios. The number of subpopulations $N_{k} \quad 401$ results from the chosen temporal discretization

$$
t=\left[t_{0}, t_{1}, \ldots, t_{k-1}, t_{k}, t_{k+1}, \ldots, t_{N_{k}}\right] .
$$

Here, an equidistant grid with $\left(t_{k}-t_{k-1}\right)=0.5 t u$ and $N_{k}=2000$ was used. ${ }^{403}$ Integral quantities (i.e. moments, overall virus release rate etc.) can be approx- ${ }^{404}$ imated by

$$
\int_{\mathbf{X}} f_{i}(\mathbf{x}) i_{c}(t, \mathbf{x}) \mathrm{d} \mathbf{x} \approx \sum_{k=1}^{N_{k}} \int_{\mathbf{X}} f_{i}(\mathbf{x}) i_{c, k}(t, \mathbf{x}) \mathrm{d} \mathbf{x} .
$$

At this point it has to be emphasized that the reformulation does in general not $\quad 406$ prevent the description of time variable environments. Each initial value problem $\quad 407$ can be solved simultaneously in this case. However, in the current example the 408 intracellular virus kinetics are decoupled from the extracellular states (i.e. the 409 single cell dynamics of the infected cells (22) do not depend on $U_{c}(t)$ and $V(t) \quad{ }_{410}$ ). Under these circumstances, the overall numerical procedure can be further ${ }^{411}$ simplified [55]. Instead of solving the full problem for the discrete reformulation, ${ }^{412}$ the single infection cycle scenario is solved only once for a normalized initial $\quad 413$ 
condition

$$
\begin{aligned}
\frac{\partial i_{c}^{*}\left(t^{*}, \mathbf{x}\right)}{\partial t^{*}} & =-\nabla_{\mathbf{x}}\left\{\mathbf{h}(\mathbf{x}) i_{c}^{*}\left(t^{*}, \mathbf{x}\right)\right\}-k_{\mathrm{cd}, i_{c}}(\mathbf{x}) i_{c}^{*}\left(t^{*}, \mathbf{x}\right) \\
i_{c}^{*}\left(t^{*}=0, \mathbf{x}\right) & =\mathcal{I}(\mathbf{x})=\mathcal{N}(\mu, \mathbf{\Sigma}) .
\end{aligned}
$$

Arbitrary integral quantities can be approximated using the moment approxi- 415 mation algorithm presented previously

$$
\int_{\mathbf{X}} f_{i}(\mathbf{x}) i_{c}^{*}\left(t^{*}, \mathbf{x}\right) \mathrm{d} \mathbf{x} \approx \sum_{\alpha=1}^{N_{\alpha}} f_{i}\left(\mathbf{x}_{\alpha}^{*}\left(t^{*}\right)\right) w_{\alpha}^{*}\left(t^{*}\right) .
$$

Afterwards each of those is multiplied with the corresponding initial condition, 417 i.e. the number of newly infected cells in the interval $\left[t_{k-1}, t_{k}\right)(41)$ to obtain ${ }_{418}$ integral approximations for the subpopulations $i_{c, k}(t, \mathbf{x})$.

$$
\begin{array}{r}
\int_{\mathbf{X}} f_{i}(\mathbf{x}) i_{c, k}(t, \mathbf{x}) \mathrm{d} \mathbf{x}=\operatorname{Inf}\left(t_{k}\right) \int_{\mathbf{X}} f_{i}(\mathbf{x}) i_{c}^{*}\left(t-t_{k}, \mathbf{x}\right) \mathrm{d} \mathbf{x} \\
\approx \operatorname{Inf}\left(t_{k}\right) \sum_{\alpha=1}^{N_{\alpha}} f_{i}\left(\mathbf{x}_{\alpha}^{*}\left(t-t_{k}\right)\right) w_{\alpha}^{*}\left(t-t_{k}\right)
\end{array}
$$

Thus, the virus dynamics (27) can be written as

$$
\begin{aligned}
\frac{\mathrm{d} V(t)}{\mathrm{d} t} & =\sum_{k=1}^{N_{k}} \int_{\mathbf{X}} r_{\mathrm{rel}}(\mathbf{x}) i_{c, k}(t, \mathbf{x}) \mathrm{d} \mathbf{x}-k_{\mathrm{inf}} U_{c}(t) V(t)-k_{\mathrm{deg}} V(t) \\
& =\sum_{k=1}^{N_{k}} \operatorname{Inf}\left(t_{k}\right) \sum_{\alpha=1}^{N_{\alpha}} r_{\mathrm{rel}}\left(\mathbf{x}_{\alpha}^{*}\left(t-t_{k}\right)\right) w_{\alpha}^{*}\left(t-t_{k}\right)-k_{\mathrm{inf}} U_{c}(t) V(t)-k_{\operatorname{deg}} V(t) .
\end{aligned}
$$

In the following, the same dependency of the cell death rate on the intracellular ${ }_{421}$ components as in the second single infection cycle scenario is assumed

$$
k_{\mathrm{cd}, i_{c}}(\mathbf{x})=k_{\mathrm{cd}, i_{c}} r_{\mathrm{rel}}(\mathbf{x})=k_{\mathrm{cd}, i_{c}} k_{5}[\text { gen }][s t r] .
$$

The overall uninfected and infected cell concentration dynamics are depicted $\quad{ }_{423}$ in Fig. 6. It can be seen, that the concentration of infected cells increases signifi- ${ }^{424}$ cantly after a certain delay of round about $300 \mathrm{tu}$. This is a direct result of a low ${ }_{425}$ initial MOI: At the begin only a low number of uninfected cells gets infected and ${ }_{426}$ 

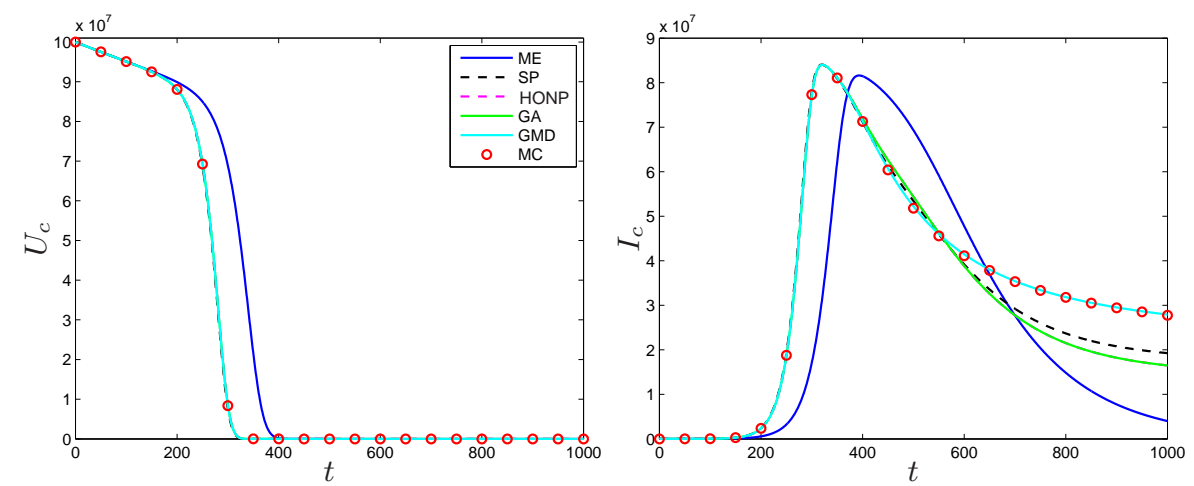

Figure 6: Multiple infection cycle scenario: overall cell dynamics for different cubatures

the infection takes a certain time to spread through the whole system. On the ${ }_{427}$ single cell scale, the viral replication mechanism and the resulting virus release $\quad 428$ rate (see Fig. 4(a)) additionally contribute to the delay.

It can be seen that all cubature formulas show nearly the same results for $\quad 430$ the uninfected cells, only the approximation which is based on a sole cubature $\quad{ }^{431}$ abscissa at the mean of the initial distribution yields significant errors. However, ${ }_{432}$ differences are obtained for the dynamics of the overall number of infected cells. $\quad 433$ Again, the mean abscissa approximation shows the worst performance. Other ${ }_{434}$ approximation approaches, based on the standard sigma points, the Gaussian 435 cubatures and the higher order non-product formula are at least sufficiently ${ }_{436}$ accurate for $t<600 t u$, but their approximation quality worsens for larger sim- ${ }_{437}$ ulation times. Again, the performance of the Gaussian mixed density approach $\quad 438$ stands out and stays very close to the reference computed with a large num- ${ }_{439}$ ber of random abscissas. These statements on the approximation accuracy are 440 also valid for the overall virus concentration dynamics which are shown in Fig. $7 \quad 441$ Excluding the mean abscissa approach, all approaches show a good performance $\quad 442$ for $t<500 t u$ but significant errors emerge for larger simulation times where $\quad{ }_{443}$ only the Gaussian mixed density approach gives accurate results. The same is ${ }^{444}$ observed for the first and second order pure moments which are depicted in 445 Fig. 8. It can be seen that the approximations based on standard sigma points, ${ }_{446}$ the Gaussian cubature or the higher order non product cubature formula are ac- $\quad 447$ 


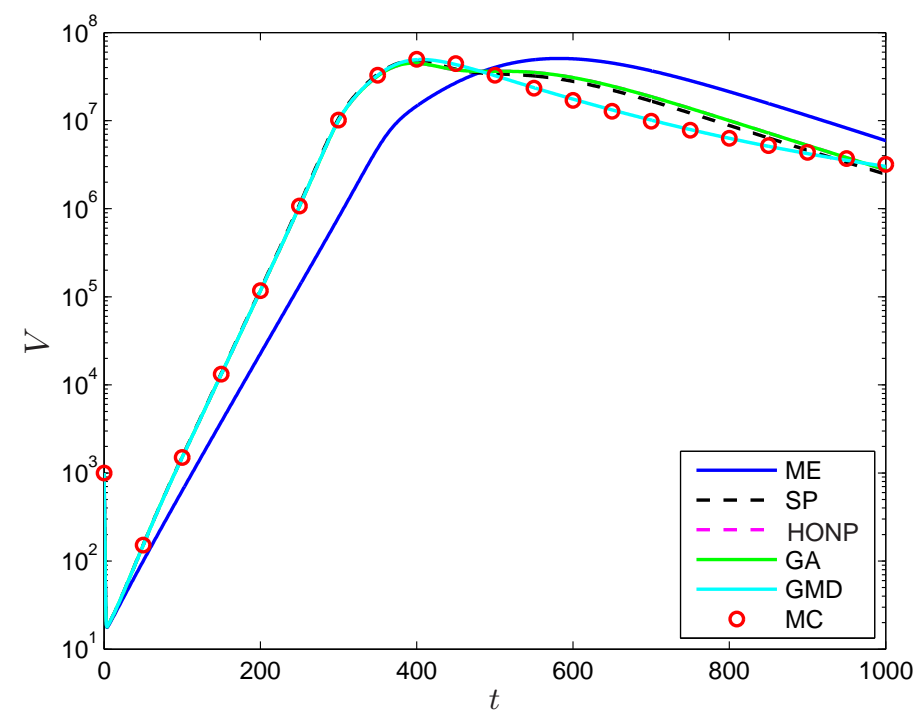

Figure 7: Multiple infection cycle scenario: Virus concentration for different cubature formulas
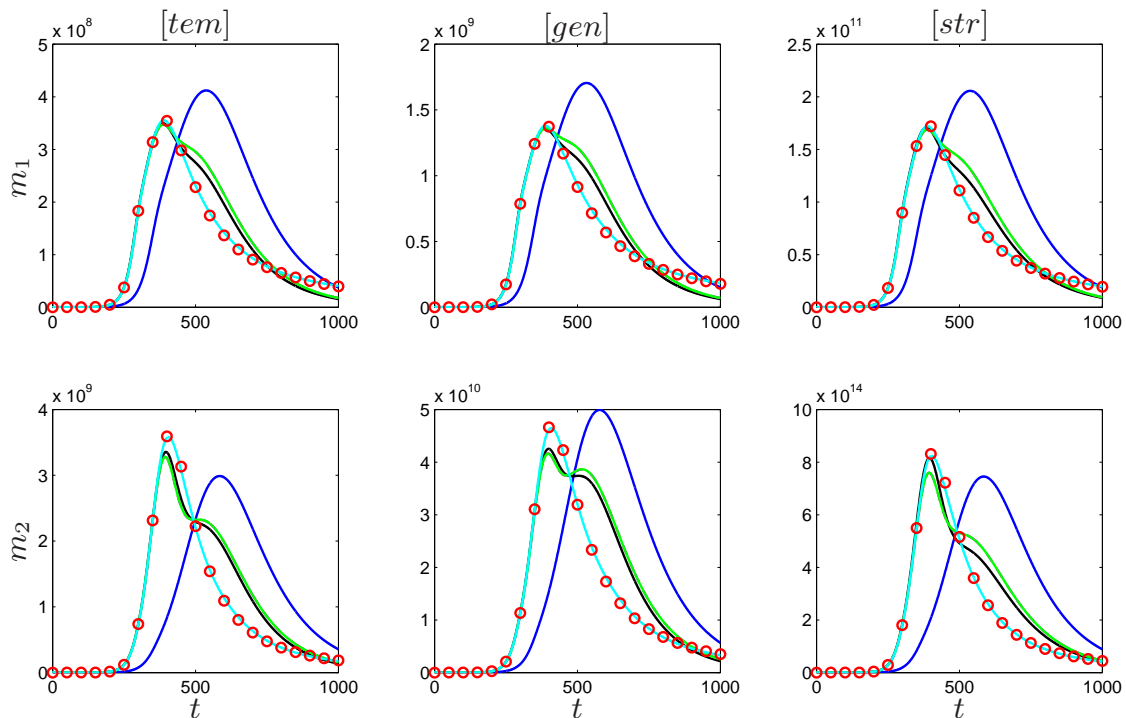

Figure 8: Multiple infection cycle: Pure moments of the distribution for different cubatures

curate up to $t \approx 400 t u$ but show significant errors for later time points. Again ${ }_{448}$ it can be seen, that the GMD stays very close to the reference solution and 449 provides an accurate approximation. 
Thus, it has been shown that a sufficient accuracy of the multi-dimensional $\quad{ }_{451}$ moments can be achieved by using GMD approach for the choice of the initial ${ }_{452}$ abscissas. This approach constitutes a direct extension of the SP approach with $\quad{ }_{453}$ which the problem of choosing a good sigma point distribution can be avoided $\quad 454$ by the cost of more abscissas. For increasing $N_{\text {GMD }}$ the overall GMD approxi- ${ }_{455}$ mation approaches the corresponding MC approximation. Thus, by successively ${ }_{456}$ increasing the number of applied GMDs, a good approximation accuracy can $\quad 457$ be achieved easily. In the current example $N_{\mathrm{GMD}}=11$ lead to an efficient ap- ${ }_{458}$ proximation accuracy.

\section{Application to a detailed model of influenza virus replication in 460 cell cultures

In a second step, the methodology proposed in this paper is applied to a ${ }_{462}$ detailed model of influenza A virus replication in mammalian cell cultures. Cell ${ }_{463}$ culture based technology has developed as a promising alternative for influenza 464 vaccine production compared to traditional processes in embryonated chicken $\quad 465$ eggs [60, 61]. Main advantage is increased flexibility which allows to respond ${ }_{466}$ rapidly to the frequently changing demands. From the practical point of view ${ }_{467}$ there is a high interest for the development of genetically engineered cell lines to $\quad 468$ establish a high yield production platform. Here, genetic modifications, e.g. by $\quad{ }_{469}$ lentiviral transduction, are used to enhance or inhibit certain steps of the viral 470 replication mechanism aiming for an increased cell specific viral production rate. $\quad 471$ However, usually not all cells are modified with the same efficiency [62]. Thus 472 a significant cell-to-cell variability with respect to the intracellular kinetics and 473 the viral production rates is expected. $\quad 474$

To analyze the impact of these variances a detailed single cell model of in- $\quad 475$ fluenza A virus replication in mammalian cell cultures can be used [24]. Therein, 476 the interactions of a large number of viral components are considered. The basic $\quad 477$ $\begin{array}{ll}\text { scheme is depicted in Fig. } 9 . & 478\end{array}$

The full set of dynamic equations and kinetic parameters according to [63] $\quad 479$ 


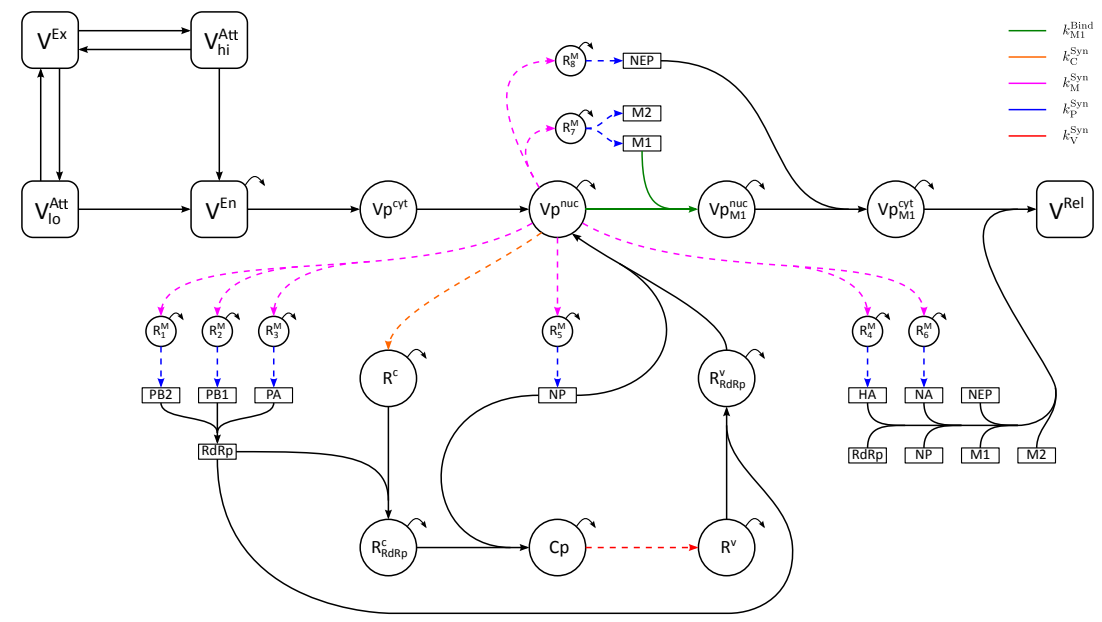

Figure 9: Basic scheme of the single cell kinetics with affected reaction steps

can be found in the supplementary information. In the following, a short overview $\quad 480$ of the process shall be given. Free virus particles $\mathrm{V}^{\mathrm{Ex}}$ attach to the cell surface ${ }_{481}$ at binding sites with either high or low affinity. Attached virus particles $\left(\mathrm{V}_{\mathrm{hi}}^{\mathrm{Att}}{ }_{482}\right.$ and $\mathrm{V}_{\mathrm{lo}}^{\text {Att }}$ ) either detach again or they are absorbed via endocytosis resulting ${ }_{483}$ in the enclosure of the virus particle $\mathrm{V}^{\mathrm{En}}$ by an intracellular endosome. After ${ }^{484}$ fusion of the viral envelope with the endosomal membrane the segmented viral $\quad 485$ genome is released into the cytoplasm in the form of eight viral ribonucleoprotein $\quad 486$ complexes (vRNPs). In Fig. 9 these vRNPs are denoted as $\mathrm{Vp}^{\text {cyt }}$. Subsequently, ${ }_{487}$ they are imported into the nucleus $\left(\mathrm{Vp}^{\text {nuc }}\right)$ where transcription into messenger $\quad 488$ RNAs (mRNAs, $\mathrm{R}_{1 \ldots 8}^{\mathrm{M}}$ ) and complementary RNAs (cRNAs, $\mathrm{R}^{\mathrm{C}}$ ) takes place. Vi- ${ }_{489}$ ral mRNAs migrate to the cytoplasm are translated into viral proteins PA, PB1, 490 PB2, HA, NP, NA, NEP, M1, and M2 of which the first three form the RNA- ${ }_{491}$ dependent RNA polymerase (RdRp). In the nucleus cRNAs are stabilized by 492 consecutive attachment of RdRp and nucleoprotein NP forming $\mathrm{cRNPs}$ (Cp). ${ }_{493}$ In a second step freshly produced cRNPs are used to synthesize new vRNA 494 molecules $\left(\mathrm{R}^{\mathrm{v}}\right)$, which are also encapsidated by RdRp and NP forming stabi- ${ }_{495}$ lized progeny vRNPs $\left(\mathrm{Vp}^{\text {nuc }}\right)$. By binding of viral matrix protein M1, vRNPs 496 are excluded from any further participation in the replication cycle $\left(\mathrm{Vp}_{\mathrm{M} 1}^{\mathrm{nuc}}\right)$. ${ }_{497}$ 
Subsequent attachment of nuclear export protein NEP initiates the export to 498 the cytoplasm $\left(\mathrm{Vp}_{\mathrm{M} 1}^{\mathrm{cyt}}\right)$. Eventually, all necessary vRNP complexes and viral pro- ${ }^{499}$ teins assemble at the cell membrane to form progeny virions that bud from the 500 surface into the surrounding medium as newly released virus particles $\left(\mathrm{V}^{\mathrm{Rel}}\right) . \quad{ }_{501}$

The single cell description was used to come up with a bottom-up population $\quad 502$ balance model [44]. The latter can be viewed as direct extension of a previously 503 proposed age-structured model of the process [63]. It enables the combination 504 of detailed description of the viral replication mechanisms with the overall in- 505 teraction of virus, infected and uninfected cells on the cell population level. In 506 this contribution, recent numerical results will be presented briefly. For details 507 on implementation we refer to [44]. 508

Five significant steps of the viral replication cycle, i.e. the production of viral 509 proteins, as well as viral mRNA, cRNA, vRNA synthesis and the binding rate of 510 M1, were assumed to be target of genetic modifications aiming at an increased 511 maximum of virus concentration and a minimum peak time. The modified in- 512 tracellular reactions are highlighted in the single cell reaction scheme in Fig. 9. 513 In a pragmatic sensitivity analysis $3^{5}$ different parameter sets of the five rates 514 were obtained to investigate the impact of up- or downregulation by means of 515 increasing or decreasing the corresponding rates or keeping them at the original 516 values. Therein, it was assumed that the rates are the same for all cells. For that 517 reason each of the $3^{5}$ candidate parameter sets was used to simulate the dynamic $\quad 518$ behaviour of a homogeneous cell population with the proposed approximate mo- $\quad 519$ ment method using only one abscissa which is located at the exact parameter $\quad 520$ values. Here, it should be noted that the cell population is homogeneous re- ${ }_{521}$ garding to the modified parameters, but a variability in the stage of infection $\quad 522$ caused by low initial seed virus concentration was considered further. Simu- 523 lation results of each parameter set were screened for an increased maximum 524 virus production and a minimum peak time to evaluate the up-/downregulation $\quad 525$ combination. Concerning that criteria, the combination of upregulated synthe- 526 sis rates of mRNA, vRNA and proteins together with a downregulation of the ${ }_{527}$ binding rate of M1 to progeny vRNPs and an unchanged parameter for the ${ }_{528}$ 


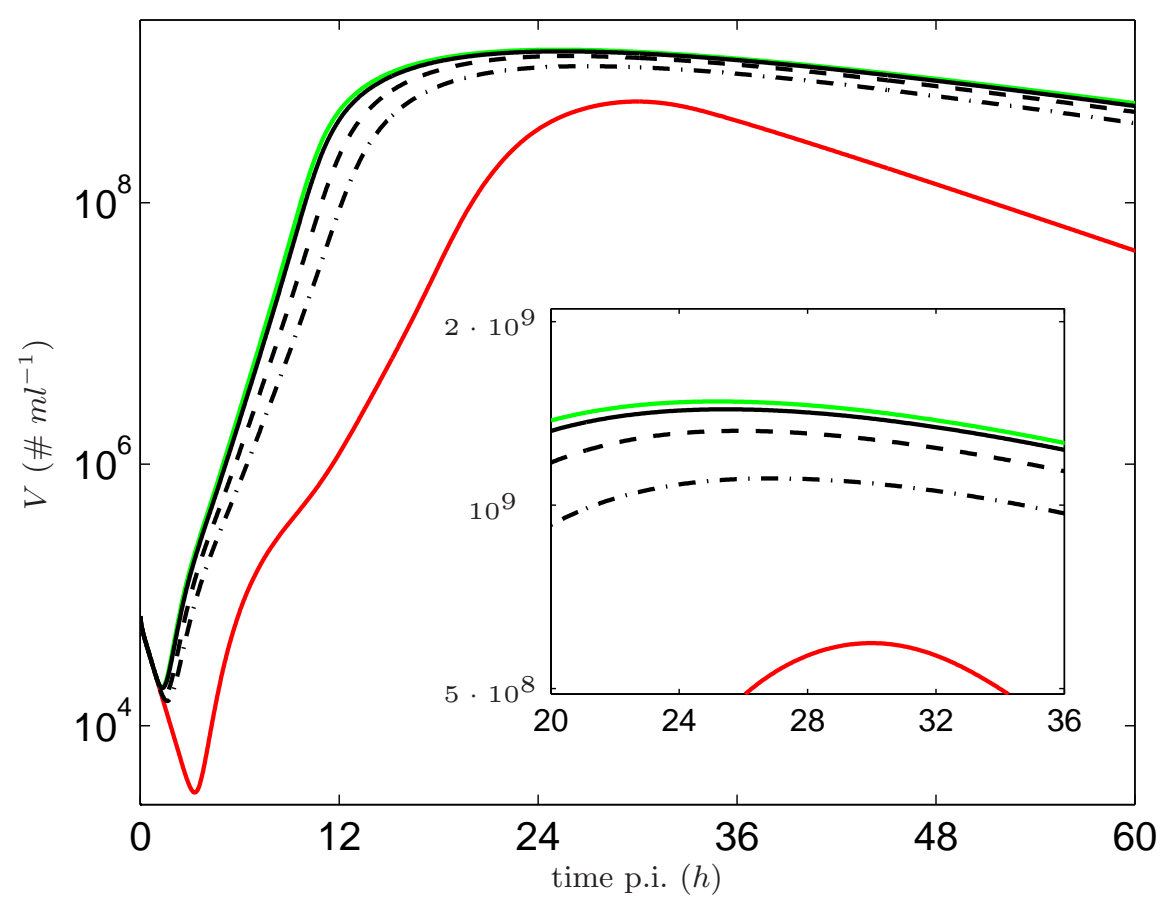

Figure 10: Virus dynamics for detailed PBE for influenza virus replication in mammalian cell cultures; simulation results for homogeneous unmodified cell population (red), homogeneous modified cell population (green) and heterogeneous cell population (black, increasing cellular variance: solid $\rightarrow$ dashed $\rightarrow$ dash-dotted)

synthesis of cRNA represents the best parameter set (see also $\mu_{5}$ in Eq. 50). ${ }_{529}$ The simulation result with this best combination is depiced in Fig. 10 (green 530 line). The red line in Fig. 10 represents the unmodified cell line dynamics for ${ }_{531}$ comparison. It can be seen that both aims, increase of the maximum virus yield 532 and reduction of peak time are achieved (see also Tab. 2). Investigated cellu- 533 lar modifications can be achieved by biotechnological methods, e.g. lentiviral 534 transduction $[64,65]$. However, in general not all cells are modified with the 535 same efficiency. This leads to cellular heterogeneity and can be accounted for by $\quad 536$ assuming that kinetic parameters are not uniform for the whole cell population $\quad 537$ but appear distributed. In consequence, different efficiencies of the genetic mod- 538 ifications can be mapped to broadening parameter distributions. The cell-to-cell 539 variability resulting genetic modifications is represented by a weighted sum of 540 
Table 2: Maximum virus yield and peak time for modified and unmodified cell dynamics

\begin{tabular}{ccc} 
& $\max (V)\left(\# m l^{-1}\right)$ & peak time $(h)$ \\
\hline unmodified & $5.9410^{8}$ & 30 \\
homogeneous modification & $1.4710^{9}$ & 25.2 \\
scenario $I$ & $1.4410^{9}$ & 25.5 \\
scenario $I I$ & $1.3210^{9}$ & 25.9 \\
scenario $I I I$ & $1.1110^{9}$ & 26.2 \\
\hline
\end{tabular}

five logarithmic Gaussians to obtain a well-shaped distribution. As five reaction $\quad 541$ rates are considered, those Gaussians are five dimensional

$$
\mathbf{k}=\sum_{l=1}^{5} a_{l} \mu_{\mathbf{l}} \cdot e^{\mathcal{N}\left(\mathbf{0}, \operatorname{diag}\left(\sigma_{\mathbf{l}}\right)\right)}
$$

The mean vectors of the distributions $\mu_{1}$ are logarithmically distributed between $\quad 543$ the nominal parameter vector $\mu_{1}$ and the best combination $\mu_{5}$

$$
\begin{aligned}
& \mu_{1}=\left[k_{C}^{S y n}, k_{M}^{S y n}, k_{P}^{S y n}, k_{V}^{S y n}, k_{M 1}^{\text {Bind }}\right]^{T}, \\
& \mu_{5}=\left[k_{C}^{S y n}, 5 k_{M}^{S y n}, 5 k_{P}^{S y n}, 5 k_{V}^{S y n}, 0.2 k_{M 1}^{\text {Bind }}\right]^{T} .
\end{aligned}
$$

The variances are chosen as $\sigma_{l}=0.05$ to obtain a well-shaped distribution. The ${ }^{545}$ influence of different modification efficiencies was analyzed for highly efficient, 546 mid-range or broad distributions of the parameter rates based on publications $\quad 547$ on transduction methods $[64,65]$. The three different scenarios are realized using $\quad 548$ different values of the weighting parameters $a_{l}$

$$
\begin{aligned}
\mathbf{a}_{I} & =[0.01,0.03,0.04,0.06,0.86], \\
\mathbf{a}_{I I} & =[0.04,0.12,0.19,0.27,0.38], \\
\mathbf{a}_{I I I} & =[0.20,0.20,0.20,0.20,0.20] .
\end{aligned}
$$

For each scenario, the moment dynamics were approximated with our proposed 550 technique applying the GMD approach. Different numbers of Gaussian distribu- 551 tions were evaluated against a Monte-Carlo approximation with $N_{\alpha, \mathrm{MC}}=10^{4} \quad{ }^{552}$ 


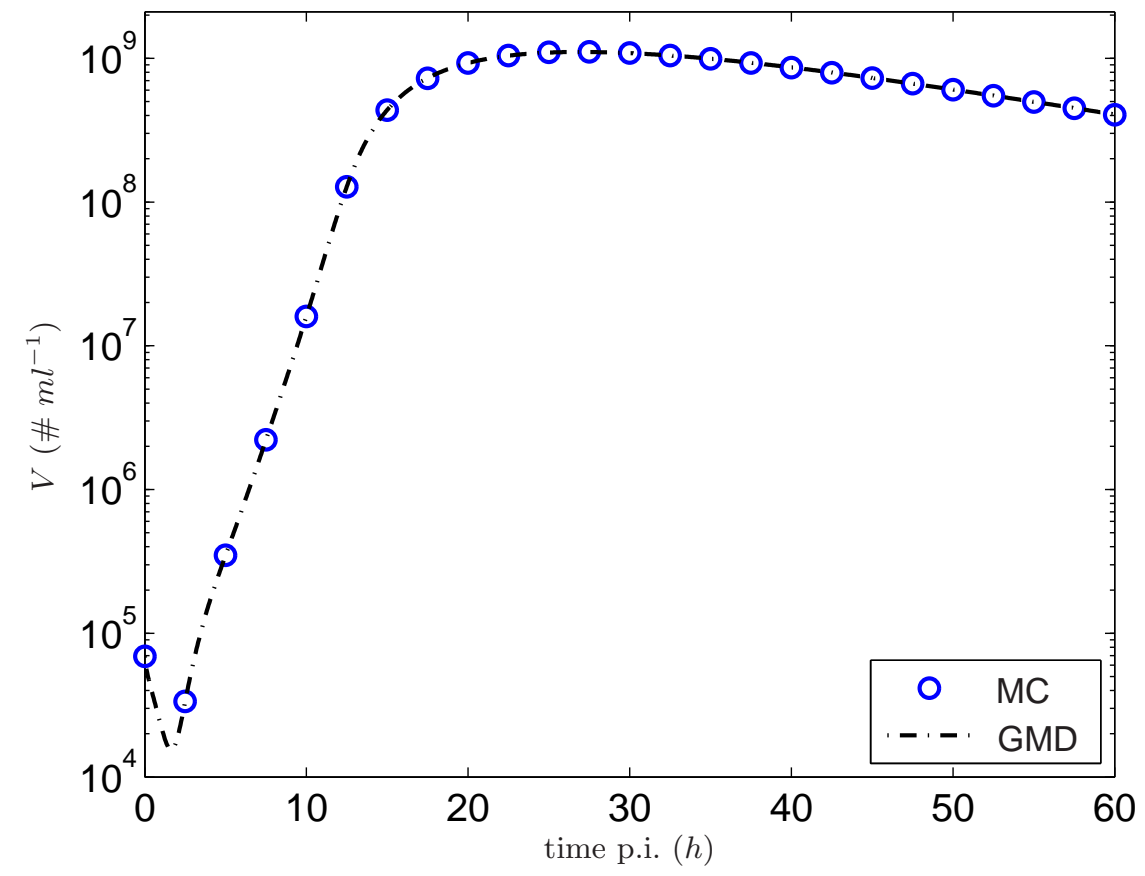

Figure 11: Comparison of virus dynamics for third scenario; proposed GMD approach using $N_{\alpha, \mathrm{GMD}}=150$ abscissas (dash-dotted) and reference $M C$-approximation using $N_{\alpha, \mathrm{MC}}=10^{4}$ (blue)

and $N_{\text {GMD }}=15$ was chosen for a good approximation accuracy (see Fig. 11). $\quad{ }_{553}$ Thus, the overall number of abscissas is given by $N_{\alpha, \mathrm{GMD}}=N_{\mathrm{SP}} N_{\mathrm{GMD}}=150 . \quad{ }^{554}$ It can be seen in Fig. 10, that an increasing variance is attended by a reduced 555 maximum virus yield and an increasing peak time. However, in comparison to 556 the unmodified cell line even for the worst case scenario an significant increase $\quad 557$ in the maximum virus concentration is obtained for the assumed genetic modifi- 558 cations (see Tab 2). Thus it can be concluded, that the above modifications are 559 a promising candidate for improving influenza vaccine production processes. $\quad 560$

\section{Conclusion}

Population balance modeling offers a suitable framework to characterize the $\quad 562$ dynamics of nonuniform particle ensembles as they are found in many applica- 563 
tions from chemical and biotechnological process systems. In particular for the 564 latter, a large number cellular properties has to be accounted for. The resulting 565 multi-dimensional PBEs can be solved numerically using approximate moment $\quad 566$ methods. 567

In this manuscript we presented an efficient method for the approximate com- $\quad 568$ putation of moments for this type of models, which is based on the DQMOM. ${ }_{569}$ Analytical solutions for the dynamics of weights and abscissas are derived and, 570 thus, the subsequent numerical solution of linear equation systems can be omit- 571 ted . Furthermore, a sophisticated choice of the abscissas with a sigma point 572 cubature rule ensures a limited numerical effort. In contrast to Gaussian cuba- 573 tures which scale exponentially with the dimension of the problem, here, the 574 numerically effort increases linearly. The algorithm is equivalent to our recently 575 presented technique [41]. 576

The algorithm is evaluated for a benchmark problem describing virus repli- $\quad 577$ cation in cell cultures. Therefore different cubature approaches are compared to $\quad 578$ a Monte Carlo evaluation. The presented approach shows good accuracy for all 579 implemented formulas in a first test case where cell death does not depend on the $\quad 580$ intracellular state. It is shown that the performance can be further improved by 581 using higher order non cubature and a Gaussian mixed density approach, respec- 582 tively. In contrast to other applied cubature rules, the latter also gives excellent $\quad 583$ results if cell death depends nonlinearly on the intracellular composition. The ${ }_{584}$ corresponding numerical effort increases in comparison to the standard sigma 585 point formula but is still smaller compared to Gaussian cubatures. 586

In addition, application is briefly shown for a detailed PBE describing in- ${ }^{587}$ fluenza virus replication. The presented technique is applied to analyze on the 588 overall virus dynamics when using genetically modified cells. It was shown that 589 even for a large degree of cellular variance, a significant increase in the maximum $\quad 590$ virus titer can be obtained. The results suggest, that genetic modifications may 591 be a suitable tool to come up with a high yield cell line for influenza vaccine 592 production. 593

In the future, focus will be on further application of the technique for model 594 
based analysis of the effects of cellular variance when using genetically modified 595 cell lines to overcome limitations in vaccine production processes. Furthermore, 596 extension of the algorithm on bio systems with non negligible cell division is 597 worthwhile. In addition, the method may be extended to problems with non 598 negligible spatial gradients and to agglomeration processes. An alternative ap- 599 proach for the latter is the moving pivot method [66] which is also based on 600 some moving reference points like the presented approach. 601

Although focus in this manuscript was on bio systems, the algorithm can 602 also be applied to other processes that can be described by multi-dimensional 603 population balance equations, e.g. shape evolution in crystallization processes. $\quad 604$

\section{Acknowledgements}

Stefanie Duvigneau and Robert Dürr are also affiliated to the "International 606 Max Planck Research School for Advanced Methods in Process and System 607 Engineering", which is located in Magdeburg. 608

The authors gratefully acknowledge the funding of this work by the German 609 Federal Ministry of Science and Education [Bundesministerium für Bildung und 610 Forschung (BMBF)] as part of the "e:Bio" - project "CellSys" (grant number 611 $0316189 \mathrm{~A})$.

\section{Appendix}

Evaluation of weight and abscissa dynamics for arbitrary moments. For $P=614$ 0 and $N_{d}=2(8)$ reduces to

$$
\begin{gathered}
\sum_{\alpha=1}^{N_{\alpha}}\left[a_{\alpha}\left(1-l_{1}-l_{2}\right) x_{1, \alpha}^{l_{1}} x_{2, \alpha}^{l_{2}}+b_{1, \alpha} l_{1} x_{1, \alpha}^{l_{1}-1} x_{2, \alpha}^{l_{2}}+b_{2, \alpha} l_{2} x_{1, \alpha}^{l_{1}} x_{2, \alpha}^{l_{2}-1}\right] \\
=\sum_{N_{\alpha}}^{\alpha=1} w_{\alpha}\left[l_{1} x_{1, \alpha}^{l_{1}-1} x_{2, \alpha}^{l_{2}} G_{1, \alpha}+l_{2} x_{1, \alpha}^{l_{1}} x_{2, \alpha}^{l_{2}-1} G_{2, \alpha}-D_{\alpha} x_{1, \alpha}^{l_{1}} x_{2, \alpha}^{l_{2}}\right] .
\end{gathered}
$$

In the next step the analytic expressions for the dynamics of weights and abscis- ${ }_{616}$ sas can be derived by comparison of the coefficients for zeroth and first order 617 


$$
\begin{gathered}
a_{\alpha}=-w_{\alpha} D_{\alpha} \\
b_{1, \alpha}=w_{\alpha}\left(G_{1, \alpha}-x_{1, \alpha} D_{\alpha}\right), \quad b_{2, \alpha}=w_{\alpha}\left(G_{2, \alpha}-x_{2, \alpha} D_{\alpha}\right) .
\end{gathered}
$$

These are inserted to (52) resulting in

$$
\begin{aligned}
& \sum_{\alpha=1}^{N_{\alpha}}\left[-w_{\alpha} D_{\alpha}\left(1-l_{1}-l_{2}\right) x_{1, \alpha}^{l_{1}} x_{2, \alpha}^{l_{2}}\right. \\
& \left.+w_{\alpha}\left(G_{1, \alpha}-x_{1_{\alpha}} D_{\alpha}\right) l_{1} x_{1, \alpha}^{l_{1}-1} x_{2, \alpha}^{l_{2}}+w_{\alpha}\left(G_{2, \alpha}-x_{2_{\alpha}} D_{\alpha}\right) l_{2} x_{1, \alpha}^{l_{1}} x_{2, \alpha}^{l_{2}-1}\right] \\
= & \sum_{\alpha=1}^{N_{\alpha}} w_{\alpha}\left[l_{1} x_{1, \alpha}^{l_{1}-1} x_{2, \alpha}^{l_{2}} G_{1, \alpha}+l_{2} x_{1, \alpha}^{l_{1}} x_{2, \alpha}^{l_{2}-1} G_{2, \alpha}-D_{\alpha} x_{1, \alpha}^{l_{1}} x_{2, \alpha}^{l_{2}}\right] .
\end{aligned}
$$

The left hand side expression can be rearranged and condensed to

$\sum_{\alpha=1}^{N_{\alpha}} w_{\alpha}\left[l_{1} x_{1, \alpha}^{l_{1}-1} x_{2, \alpha}^{l_{2}} G_{1, \alpha}+l_{2} x_{1, \alpha}^{l_{1}} x_{2, \alpha}^{l_{2}-1} G_{2, \alpha}+x_{1, \alpha}^{l_{1}} x_{2, \alpha}^{l_{2}} D_{\alpha}\left(-1+l_{1}+l_{2}-l_{1}-l_{2}\right)\right]$

which equals the right hand side of (52).

621 


\section{Notation}

$\begin{array}{ll}A & \text { matrix } \\ \mathbf{a} & \text { dynamics of weights } \\ \mathbf{b} & \text { dynamics of abscissas } \\ \mathbf{c} & \text { continuous phase state vector } \\ D & \text { death rate } \\ \mathbf{D}_{\mathbf{c}} & \text { medium exchange rate vector } \\ d & \text { dimension of } \mathbf{x} \\ \mathbf{F} & \text { exchange rate between dispersed and continuous phase } \\ \mathbf{G} & \text { vector of growth rates } \\ g & \text { growth rate } \\ i_{c} & \text { infected cell number density distribution } \\ k & \text { index } \\ k_{\circ} & \text { parameter } \\ l & \text { index } \\ m_{l_{1}, \ldots, l_{d}}(t) & \text { moment of order } l_{1}, \ldots, l_{d} \text { with respect to } n \\ \mathcal{N}(\mu, \boldsymbol{\Sigma}) & \text { Gaussian normal distribution } \\ n(t, \mathbf{x}), \widetilde{n}(t, \mathbf{x}) & \text { number density distribution } \\ n_{0} & \text { initial number density distribution } \\ \mathrm{N} & \text { overall number of particles } \\ N_{\alpha} & \text { number of abscissas } \\ N_{d} & \text { number of dimensions } \\ P & \text { particle production rate } \\ \mathbf{P} & \text { integral coupling rate vector } \\ t & \text { concentration of uninfected cells } \\ U_{c} & \text { extracellular virus concentration } \\ V & \text { set of cubature weights } \\ \mathcal{X} & w_{\alpha}\end{array}$


$\mathbf{x}_{e}$

$\mathbf{x}_{i}$

$\mathbf{x}_{\alpha}$

$[0]$ vector of external coordinates

vector of internal coordinates

cubature abscissa

concentration of intracellular compound o

Greek Symbols

$\alpha$ index

$\widetilde{\alpha}$ tuning factor

$\beta \quad$ tuning factor

$\kappa \quad$ tuning factor

$\lambda$ tuning factor

$\mu \quad$ mean value vector

$\Sigma \quad$ covariance matrix

Abbreviations

$D Q M O M \quad$ direct quadrature method of moments

$O D E \quad$ ordinary differential equation

$P B E \quad$ population balance equation

$P D E \quad$ partial differential equation

QMOM quadrature method of moments 


\section{References}

[1] M. Peglow, J. Kumar, R. Hampel, E. Tsotsas, S. Heinrich, Towards a Complete Population Balance Model for Fluidized-Bed Spray Agglomeration, Drying Technology 25 (7-8) (2007) 1321-1329.

[2] A. Vreman, C. van Lare, M. Hounslow, A basic population balance model for fluid bed spray granulation, Chemical Engineering Science 64 (21) (2009) 4389-4398.

[3] R. Radichkov, T. Müller, A. Kienle, S. Heinrich, M. Peglow, L. Mörl, A numerical bifurcation analysis of continuous fluidized bed spray granulation with external product classification, Chemical Engineering and Processing: Process Intensification 45 (10) (2006) 826 - 837.

[4] C. Borchert, K. Sundmacher, Morphology evolution of crystal populations: Modeling and observation analysis, Chemical Engineering Science 70 (0) (2012) $87-98$.

[5] J. J. Liu, C. Y. Ma, Y. D. Hu, X. Z. Wang, Modelling protein crystallisation using morphological population balance models, Chemical Engineering Research and Design 88 (4) (2010) 437 - 446.

[6] D. L. Ma, D. K. Tafti, R. D. Braatz, Optimal control and simulation of multidimensional crystallization processes, Computers \& Chemical Engineering 26 (78) (2002) 1103-1116.

[7] N. Hampel, A. Bück, M. Peglow, E. Tsotsas, Continuous pellet coating in a Wurster fluidized bed process, Chemical Engineering Science 86 (2013) 87-98, doi:http://dx.doi.org/10.1016/j.ces.2012.05.034.

[8] G. Y. Zhu, A. Zamamiri, M. A. Henson, M. A. Hjortso, Model predictive control of continuous yeast bioreactors using cell population balance models, Chemical Engineering Science 55 (24) (2000) 6155-6167. 
[9] Y. Zhang, A. M. Zamamiri, M. A. Henson, M. A. Hjortso, Cell population models for bifurcation analysis and nonlinear control of continuous yeast bioreactors, Journal of Process Control 12 (6) (2002) 721-734.

[10] P. Mhaskar, M. A. Hjortso, M. A. Henson, Cell population modeling and parameter estimation for continuous cultures of Saccharomyces cerevisiae, Biotechnology Progress 18 (5) (2002) 1010-1026.

[11] A. Franz, H.-S. Song, D. Ramkrishna, A. Kienle, Experimental and theoretical analysis of poly(-hydroxybutyrate) formation and consumption in Ralstonia eutropha, Biochemical Engineering Journal 55 (1) (2011) 49 58.

[12] A. Franz, R. Dürr, A. Kienle, Population Balance Modeling of Biopolymer Production in Cellular Systems, in: Proceedings to 19th IFAC WC - Cape Town, 1705-1710, 2014.

[13] J. Schulze-Horsel, Y. Genzel, U. Reichl, Flow cytometric monitoring of influenza A virus infection in MDCK cells during vaccine production, BMC Biotechnology 8 (45).

[14] T. Müller, R. Dürr, B. Isken, J. Schulze-Horsel, U. Reichl, A. Kienle, Distributed modeling of human influenza a virus-host cell interactions during vaccine production, Biotechnology and Bioengineering 110 (8) (2013) 22522266.

[15] S. Müller, H. Harms, T. Bley, Origin and analysis of microbial population heterogeneity in bioprocesses, Current Opinion in Biotechnology 21 (1) (2010) $100-113$.

[16] V. Chickarmane, C. Toein, U. A. Nuber, H. M. Sauro, C. Peterson, Transcriptional Dynamics of the Embryonic Stem Cell Switch, PLoS Computational Biology 2 (9) (2006) e123.

[17] M. Herberg, I. Roeder, Computational modelling of embryonic stem-cell fate control, Development 142 (13) (2015) 2250-2260. 
[18] T. Eissing, H. Conzelmann, E. D. Gilles, F. Allgöwer, E. Bullinger, P. Scheurich, Bistability analyses of a caspase activation model for receptorinduced apoptosis, Journal of Biological Chemistry 279 (35) (2004) 36892 36897.

[19] H. Hulburt, S. Katz, Some problems in particle technology: A statistical mechanical formulation, Chemical Engineering Science 19 (8) (1964) 555 574 .

[20] A. G. Fredrickson, D. Ramkrishna, H. M. Tsuchiya, Statistics and dynamics of procaryotic cell populations, Mathematical Biosciences 1 (3) (1967) 327374.

[21] D. Ramkrishna, Population Balances: Theory and Applications to Particulate Systems in Engineering, Academic Press, San Diego, 2000.

[22] D. Ramkrishna, M. R. Singh, Population Balance Modeling: Current Status and Future Prospects, Annual Review of Chemical and Biomolecular Engineering 5 (2014) 123-146.

[23] R. Dürr, T. Müller, B. Isken, J. Schulze-Horsel, U. Reichl, A. Kienle, Distributed Modeling and Parameter Estimation of Influenza Virus Replication During Vaccine Production, in: Proceedings to 7th Vienna International Conference on Mathematical Modelling - MATHMOD 2012, 320-325, 2012.

[24] S. F. Heldt, T. Frensing, U. Reichl, Modeling the intracellular dynamics of influenza virus replication to understand the control of viral RNA synthesis, Journal of Virology 86 (15) (2012) 7806-7817.

[25] N. V. Mantzaris, P. Daoutidis, F. Srienc, Numerical solution of multivariable cell population balance models: I. Finite difference methods, Computers \& Chemical Engineering 25 (11-12) (2001) 1411 - 1440. 
[26] N. V. Mantzaris, P. Daoutidis, F. Srienc, Numerical solution of multivariable cell population balance models. III. Finite element methods, Computers \& Chemical Engineering 25 (11-12) (2001) 1463 - 1481.

[27] V. John, C. Suciu, Direct discretizations of bi-variate population balance systems with finite difference schemes of different order, Chemical Engineering Science 106 (0) (2014) 39 - 52.

[28] M. N. Nandanwar, S. Kumar, A new discretization of space for the solution of multi-dimensional population balance equations, Chemical Engineering Science 63 (8) (2008) $2198-2210$.

[29] R. Gunawan, I. Fusman, R. D. Braatz, Parallel high-resolution finite volume simulation of particulate processes, AIChE Journal 54 (6) (2008) 14491458.

[30] L. N. Trefethen, Spectral Methods in MATLAB, SIAM, ISBN 0-89871-465$6,2001$.

[31] A. Bück, G. Klaunick, J. Kumar, M. Peglow, E. Tsotsas, Numerical Simulation of Particulate Processes for Control and Estimation by Spectral Methods, AIChE Journal 83 (2012) 2309-2319.

[32] V. John, I. Angelov, A. A. Öncül, D. Thvenin, Techniques for the reconstruction of a distribution from a finite number of its moments, Chemical Engineering Science 62 (11) (2007) 2890 - 2904.

[33] R. McGraw, Description of aerosol dynamics by the quadrature method of moments, Aerosol Science and Technology 27 (2) (1997) 255-265.

[34] E. Aamir, Z. K. Nagy, C. D. Rielly, T. Kleinert, B. Judat, Combined Quadrature Method of Moments and Method of Characteristics Approach for Efficient Solution of Population Balance Models for Dynamic Modeling and Crystal Size Distribution Control of Crystallization Processes, Industrial \& Engineering Chemistry Research 48 (18) (2009) 8575-8584. 
[35] D. Wright, R. McGraw, D. Rosner, Bivariate Extension of the Quadrature Method of Moments for Modeling Simultaneous Coagulation and Sintering of Particle Populations, Journal of Colloid and Interface Science 236 (2) (2001) $242-251$.

[36] C. Yoon, R. McGraw, Representation of generally mixed multivariate aerosols by the quadrature method of moments: I. Statistical foundation, Journal of Aerosol Science 35 (5) (2004) $561-576$.

[37] C. Yoon, R. McGraw, Representation of generally mixed multivariate aerosols by the quadrature method of moments: II. Aerosol dynamics, Journal of Aerosol Science 35 (5) (2004) 577 - 598.

[38] A. Buffo, D. L. Marchisio, Modeling and simulation of turbulent polydisperse gas-liquid systems via the generalized population balance equation, Reviews in Chemical Engineering 30 (1) (2014) 73-126.

[39] D. L. Marchisio, R. O. Fox, Solution of population balance equations using the direct quadrature method of moments, Journal of Aerosol Science 36 (1) (2005) $43-73$.

[40] A. Zucca, D. L. Marchisio, M. Vanni, A. A. Barresi, Validation of bivariate DQMOM for nanoparticle processes simulation, AIChE Journal 53 (4) (2007) 918-931.

[41] R. Dürr, A. Kienle, An Efficient Method for Calculating the Moments of Multidimensional Growth Processes in Population Balance Systems, The Canadian Journal of Chemical Engineering 92 (2014) 2088-2097.

[42] J. Villadsen, J. Nielsen, N. Liden, Bioreaction Engineering Principles, Springer Science \& Business Media, Heidelberg, 3 edn., 2011.

[43] D. L. Marchisio, Quadrature Method of Moments for Poly-Disperse Flows, in: D. L. Marchisio, R. O. Fox (Eds.), Multiphase Reacting Flows: Modelling and Simulation, vol. 492, Springer Vienna, 41-77, 2007. 
[44] R. Dürr, Parameter estimation and method of moments for multi dimensional population balance equations with application to vaccine production processes, Ph.D. thesis, Otto-von-Guericke University Magdeburg, 2016, submitted.

[45] H.-K. Rhee, R. Aris, N. R. Amundson, First-Order Partial Differential Equations, Volume 1: Theory and Application of Single Equations, Dover Publications, Inc., Mineola, New York, 1986.

[46] P. J. Davis, P. Rabinowitz, Methods of Numerical Integration, Computer Science and Applied Mathematics, Academic Press, 2. ed., 4. print edn., 1994.

[47] A. Stroud, Approximate Calculation of Multiple Integrals, Prentice-Hall, Englewood Cliffs, N.J., Englewood Cliffs, N.J., 1971.

[48] U. Lerner, Hybrid Bayesian Networks for Reasoning about Complex Systems, Ph.D. thesis, Stanford University, 2002.

[49] S. J. Julier, J. K. Uhlmann, A Consistent, Debiased Method for Converting Between Polar and Cartesian Coordinate Systems, in: In The Proceedings of AeroSense: The 11th International Symposium on Aerospace/Defense Sensing, Simulation and Controls, 110-121, 1997.

[50] R. van der Merwe, Sigma-Point Kalman Filters for Probablilistic Inference in Dynamic State-Space Models, Ph.D. thesis, Oregon Health \& Science University, 2004.

[51] S. Julier, J. Uhlmann, Unscented filtering and nonlinear estimation, Proceedings of the IEEE 92 (3) (2004) $401-422$.

[52] R. Schenkendorf, Optimal experimental design for parameter identification and model selection, Ph.D. thesis, Otto-von-Guericke University Magdeburg, 2014. 
[53] N. Rossner, T. Heine, R. King, Quality-by-Design using a Gaussian Mixture Density Approximation of Biological Uncertainties, in: Proceedings of the 11th International Symposium on Computer Applications in Biotechnology, vol. 11, 7-12, 2010.

[54] E. L. Haseltine, J. B. Rawlings, J. Yin, Dynamics of viral infections: incorporating both the intracellular and extracellular levels, Computers \& Chemical Engineering 29 (3) (2005) 675 - 686.

[55] E. L. Haseltine, J. Yin, J. B. Rawlings, Implications of Decoupling the Intracellular and Extracellular Levels in Multi-Level Models of Virus Growth, Biotechnology and Bioengineering 101 (4) (2008) 811-820.

[56] R. Dürr, T. Müller, A. Kienle, Efficient DQMOM for Multivariate Population Balance Equations and Application to Virus Replication in Cell Cultures, in: 8th Vienna International Conference on Mathematical Modelling, $29-34,2015$.

[57] E. Sherer, R. Hannemann, A. Rundell, D. Ramkrishna, Application of stochastic equations of population balances to sterilization processes, Chemical Engineering Science 64 (4) (2009) 764-774.

[58] A. Reinhold, H. Briesen, High dimensional population balances for the growth of faceted crystals: Combining Monte Carlo integral estimates and the method of characteristics, Chemical Engineering Science 127 (2015) 220-229.

[59] T. Eissing, M. Chaves, F. Allgöwer, Live and let die-A systems biology view on cell death, Computers \& Chemical Engineering 33 (3) (2009) 583-589.

[60] J. E. Nichols, J. W. LeDuc, Influenza, in: A. D. T. Barrett, L. R. Stanberry (Eds.), Vaccines for biodefense and emerging and neglected Diseases, Academic Press, London, 497-525, 2009.

[61] Y. Genzel, U. Reichl, Continuous cell lines as a production system for influenza vaccines, Expert Rev Vaccines 8 (12) (2009) 1681-1692. 
[62] F. Bushman, M. Lewinski, A. Ciuffi, S. Barr, J. Leipzig, S. Hannenhalli, C. Hoffmann, Genome-wide analysis of retroviral DNA integration, Nature Reviews Microbiology 3 (11) (2005) 848-858.

[63] F. S. Heldt, T. Frensing, A. Pflugmacher, R. Gröpler, B. Peschel, U. Reichl, Multiscale Modeling of Influenza A Virus Infection Supports the Development of Direct-Acting Antivirals, PLoS Computational Biology 9 (11).

[64] S. A. Stewart, D. M. Dykxhoorn, D. Palliser, H. Mizuno, E. Y. Yu, D. S. An, D. M. Sabatini, I. S. Y. Chen, W. C. Hahn, P. A. Sharp, R. A. Weinberg, C. D. Novina, Lentivirus-delivered stable gene silencing by RNAi in primary cells, RNA (New York, N.Y.) 9 (4) (2003) 493-501.

[65] B. R. McNaughton, J. J. Cronican, D. B. Thompson, D. R. Liu, Mammalian cell penetration, siRNA transfection, and DNA transfection by supercharged proteins, Proceedings of the National Academy of Sciences of the United States of America 106 (15) (2009) 6111-6116.

[66] S. Kumar, D. Ramkrishna, On the solution of population balance equations by discretization - II. A moving pivot technique, Chemical Engineering Science 51 (8) (1996) 1333-1342. 